\title{
Social interactions and spillovers
}

\author{
Antonio Cabrales ${ }^{\mathrm{a}}$, Antoni Calvó-Armengol ${ }^{\mathrm{b}, \mathrm{c}, 1}$, Yves Zenou ${ }^{\mathrm{d}, *}$ \\ a Universidad Carlos III de Madrid, Spain \\ b ICREA, Spain \\ ${ }^{\mathrm{c}}$ Universitat Autònoma de Barcelona, Spain \\ d Stockholm University and IFN, Sweden
}

\begin{tabular}{l} 
\\
\hline JEL classification: \\
L22 \\
L51 \\
O31 \\
O38 \\
Keywords: \\
Peer effects \\
Network formation \\
Welfare
\end{tabular}

\begin{abstract}
A B S T R A C T
The aim of this paper is to provide a tractable model where both socialization (or network formation) and productive efforts can be analyzed simultaneously. This permits a fullfledged equilibrium/welfare analysis of network formation with endogenous productive efforts and heterogeneous agents. We show that there exist two stable interior equilibria, which we can Pareto rank. The socially efficient outcome lies between these two equilibria. When the intrinsic returns to production and socialization increase, all equilibrium actions decrease at the Pareto-superior equilibrium, while they increase at the Pareto-inferior equilibrium. In both cases, the percentage change in socialization effort is higher (in absolute value) than that of the productive effort.
\end{abstract}

\section{Introduction}

Social interactions are an important aspect of everyday's life. For example, firms interact with other firms to exchange information about new technologies, individuals meet friends and researchers go to workshops and conferences. In the economics literature, social interactions are often related to particular forms of externalities, in which the actions of a reference group affect an individual's utility. The reference group depends on the context, and it typically consists of an individual's family, neighbors, friends or peers. Social interactions are sometimes called non-market interactions to emphasize the fact that these interactions are not regulated by the price mechanism (see, in particular, Scheinkman, 2008). As pointed out by Becker (1974) and recently emphasized by the literature on social capital, ${ }^{2}$ social interactions have a crucial importance in the determination of an individual's outcomes and, hence, on his well being. The aim of this paper is to investigate the interaction between productive effort and the creation of synergies in a tractable framework. We model this interaction

\footnotetext{
We thank the editor as well as two anonymous referees for helpful comments. We also thank Sébastien Bervoets for excellent research assistance and Daron Acemoglu, Yann Bramoullé, Luis Cabral, Glenn Ellison, Drew Fudenberg, Yannis Ioannides, Willemien Kets, George Mailath, Joan de Martí, Joel Sobel, and Fernando Vega-Redondo for their comments and seminar audiences at Universitat Autònoma de Barcelona, Universitat Pompeu Fabra, Universidad Carlos III, Università ca'Foscari di Venezia, Harvard-MIT and Yale. We gratefully acknowledge the financial support from Spain's Ministry of Science and Innovation under grants CONSOLIDER INGENIO 2010 CSD2006-0016 and ECO2009-10531. Previous running titles include: "Social Interactions and Spillovers: Incentives, Segregation, and Topology", "Efforts and Synergies in Network Formation" and "Building Socio-Economic Networks: How Many Conferences Should you Attend?".

* Corresponding author.

E-mail address: yves.zenou@ne.su.se (Y. Zenou).

1 Toni passed away in November 2007. His friendship, creativity and talent will be deeply missed.

2 Coleman (1990) and Putnam (2000) are standard references. Sobel (2002) and Durlauf (2002) offer critical surveys of this literature.
} 
in a way that allows us to characterize how agents devote resources to both activities optimally. In turn, this permits a full-fledged equilibrium/welfare analysis of individual decisions and to derive unambiguous comparative statics results. Let us describe in more detail our model.

\subsection{The environment}

Our model has two main ingredients.

First, we consider a model with local complementarities in productive investment. More precisely, spillovers are generated by paired agents and are multiplicative in own's and other's productive effort. We allow for two different sources of heterogeneity. On the one hand, agents can differ in their marginal returns to own productive effort. On the other hand, for identical levels of productive efforts, spillovers can vary with the strength of the synergistic linkage across different pairs of agents. It turns out that this payoff structure allows to exactly pin down how the level of productive effort varies with the pattern of external effects exerted on each individual, and with the idiosyncratic characteristics of the agents.

Second, we assume that agents devote a (joint) amount of resources to building synergies with others. The collection of socialization efforts determines the meeting possibilities across pairs of agents and results in a pattern of, possibly heterogeneous, bilateral interactions. The intensity of each bilateral interaction which results from this socialization process becomes a scaling factor on the local production complementarities arising between these two agents.

An innovation of our study is, precisely, that the synergistic effort is generic within a community-a scalar decision. Socializing is not equivalent, in our approach, to elaborating a nominal list of intended relationships, as in the literature on network formation surveyed by Jackson (2008). ${ }^{3}$ In other words, network formation is not the result of an earmarked socialization process.

This is realistic in many applications, particularly when networks are so large that keeping track of every participant becomes a burdensome task, or when the individuals do not yet know one another. For example, businessmen go to fairs, and researchers to conferences and workshops to present their ideas or products, to listen to those of other people's, and to meet peers in general. In order to motivate the model and to better understand its empirical implications, we provide a very concrete example of a situation captured by the model. Then, we discuss all notions and results of our paper through the lens of this example. The example is as follows. The decision makers in the model are parents. Each parent is altruistic and cares about the future educational outcome of his child. Each parent exerts two types of costly effort: productive effort with the child (i.e. doing homework with the child, doing sport activities together, driving him to different activities, and so on) and socialization effort related to education (going to parental evenings, birthday parties, or any activity that involves other parents). In this example, each parent does not directly decide with whom he interacts, but rather participates in parental activities (parental evenings, birthday parties) which can eventually lead to social interactions with other parents. These activities provide valuable information about parenting techniques, reading or study materials, school conditions and other important educational inputs. As a result of these activities, some parental and child friendships are created, which then continue producing synergies, and further directed socialization over time. For those relationships, which also have important educational implications, ours can be seen as a model of how they are created.

Our choice of a model without earmarked socialization greatly improves the tractability of the analysis. Unlike with richer models of link formation, we can resort to off-the-shelf Nash equilibrium analysis without being burdened by the extreme (combinatorial) multiplicity problems of the other models. ${ }^{4}$ As a result, we can perform a standard type of equilibrium analysis that equates marginal costs and benefits of both production and socialization. Of course, this equilibrium characterization also greatly simplifies welfare and comparative statics analyzes.

\subsection{Results}

We characterize the equilibria of the model when agents take their decision about their productive effort and their socialization effort simultaneously. We show that there are two interior equilibria and one (partially) corner equilibrium, when a sufficiently large number of individuals is implicated. The (partially) corner equilibrium where agents do not invest at all in building synergies is unstable. Instead, the two interior equilibria are stable. Existence and stability of interior equilibria are obtained when the level of cross synergies as well as the heterogeneity in individual traits are not too large, which amounts to bounding from above a compound index of both payoff parameters.

For large enough populations, equilibrium actions take a particularly simple form. Recall that agents can display different marginal returns to own productive effort. We label "individual type" the value of this marginal return at the origin. We first show that the ratios of productive as well as socialization efforts across different pairs of agents are all equal to the

\footnotetext{
3 We discuss the relationship between our model and this literature below.

4 In a typical game of network formation, players simultaneously announce all the links they wish to form with. The links that form are those that are mutually announced by both partners. The cost of creating and maintaining links are then paid. As a consequence of the large multi-dimensional strategy space, and because link creation requires the mutual consent of the two parties involved, a severe coordination problem arises. As such, the game often displays a multiplicity of Nash equilibria, and very different network geometries can arise endogenously. A partial solution to this problem can be found by allowing pair-wise or coalitional deviations, or by restricting to cooperative-like network stability notions (Jackson and Wolinsky, 1996). Jackson (2008) surveys this literature, while Calvó-Armengol and Ilkiliç (2009) derive the connections between this approach and standard game-theoretic refinements.
} 
ratio of their individual types. In other words, at equilibrium, the productive and socialization efforts for a given agent are the product of his individual type with some baseline values for the productive and socialization efforts. These baseline values, in turn, are obtained from a system of two equations with two unknowns that admits exactly two positive solutions -hence the two interior equilibria. Compared to the case of an isolated agent, these baseline values are all scaled up by a synergistic multiplier, which is homogeneous across players and which depends on a compound index of heterogeneity in the community within which they interact.

This simple equilibrium characterization has a number of interesting implications. In particular, we can show that one of the interior equilibria displays both higher socialization and productive efforts than the other, so that we can talk of a high-action and a low-action equilibrium. It also turns out that the high-action equilibrium is Pareto superior. Furthermore, the socially efficient outcome lies in between the two equilibria, so that we can effectively talk about a too-high and a too-low equilibrium.

An important question is then how an exogenous change in the returns to production and socialization affect the relative production and socialization efforts at equilibrium. In turns out that, when the returns increase, all equilibrium actions decrease at the Pareto-superior equilibrium, while they increase at the Pareto-inferior equilibrium. In both cases, the percentage change in socialization effort is higher (in absolute value) than that of the productive effort.

To summarize, we provide a simple operational model of network formation with welfare predictions and clear-cut comparative statics. In substance, we identify a "too cold" and a "too hot" equilibrium. We show that socialization is more responsive than production to exogenous shocks in the parameters.

\subsection{Relation to the literature on network formation and social interactions}

A growing literature over the last two decades has examined the problem of social network formation and its implications for economic phenomena. With respect to this literature (for literature surveys, see Vega-Redondo, 2007; Goyal, 2007; Jackson, 2008) our model, as many others in the literature, studies the effects of social interactions via its impact on production. The novelty is that we model socialization/network formation via a generic effort variable, rather than as the result of an earmarked socialization process. ${ }^{5}$

One of the main objectives of the paper is to provide a tractable framework where both socialization (or network formation) and productive efforts can be analyzed in a single model. We do this in a context of Ballester et al. (2006) framework because this is a simple model that has nice properties. Observe, however, that the insight of using non-directed socialization should carry over to other types of models. Our model also differs from that of Ioannides and Soetevent (2007) in that we can characterize the exact solutions of equilibria and analyze the comparative statics and welfare properties of our model.

The paper is organized as follows. Section 2 describes the model and introduces the baseline game as well as the replica game. Section 3 contains the equilibrium and welfare analysis. The comparative statics results are gathered in Section 4 . Section 5 discusses some empirical implications of our model while Section 6 proposes different specifications that generalizes our model. All proofs are gathered in Appendix A.

\section{The game}

\subsection{The replica game}

$N=\{1, \ldots, n\}$ is a finite set of players, and $T=\{1, \ldots, t\}$ is a finite set of types for these players. We let $n$ be a multiple of $t$, that is, $n=m t$ for some integer $m \geqslant 1$, so that there is the same number of players of each type.

More precisely, we refer to the case $n=t$ as the baseline game, and to the general case $n=m t$ as the $m$-replica of this baseline game. In an $m$-replica game, there are exactly $m$ players of each type $\tau \in T$. This replica game allows us to take limits as the population becomes large without having to specify the types of the new individuals that are added.

For each player $i \in N$, we denote by $\tau(i) \in T$ his type.

We consider a simultaneous move game of network formation (or social interactions) and investment. The returns to the investment are the sum of a private component and a synergistic component. The private returns are heterogeneous across players and depend on their type. We denote ${ }^{6}$ by $\mathbf{b}=\left(b_{1}, \ldots, b_{t}\right)$ the profile of these private returns, where $0<$ $b_{1} \leqslant b_{2} \leqslant \cdots \leqslant b_{t}$. Even though each type in the replica game has the same number of individuals, we can match any finite distribution of types in a population by adding multiple copies of an individual type. ${ }^{7}$

The synergistic returns depend on the network formed on account of individual choices, as described below.

\subsection{Network formation}

Consider some $m$-replica game, $m \geqslant 1$. Let $n=m t$.

\footnotetext{
5 See Bloch and Dutta (2009) for a model with endogenous link strength but in a standard framework of directed socialization.

6 Vectors and matrices are in bold while scalars are in normal letters.

7 With enough replications, of course.
} 
Each player $i$ selects a socialization effort, $s_{i} \geqslant 0$. Let $\mathbf{s}=\left(s_{1}, \ldots, s_{n}\right)$ be a profile of socialization efforts. Then, $i$ and $j$ interact with a link intensity given by:

$$
g_{i j}(\mathbf{s})=\rho(\mathbf{s}) s_{i} s_{j}
$$

By definition, links are symmetric, that is, $g_{i j}=g_{j i}$. We also allow for self-loops (when $i=j$ ). The total interaction intensity for a player $i$ is:

$$
g_{i}(\mathbf{s})=\sum_{j=1}^{n} g_{i j}(\mathbf{s})=\rho(\mathbf{s}) s_{i} \sum_{j=1}^{n} s_{j} .
$$

We set

$$
\rho(\mathbf{s})= \begin{cases}1 / \sum_{j=1}^{n} s_{j}, & \text { if } \mathbf{s} \neq \mathbf{0}, \\ 0, & \text { if } \mathbf{s}=\mathbf{0}\end{cases}
$$

so that $g_{i}(\mathbf{s})=s_{i}$. That is, players decide upon their total interaction intensity.

In this model, the exact identity of the interacting partner is not an object of choice. Rather, players choose an aggregate level of socialization effort. This total effort is then distributed across each and every possible bilateral interaction in proportion to the partner's socialization effort. This interaction pattern arises naturally when meetings result from casual encounters rather than from an earmarked socialization process. Using the example provided in Section $1, s_{i}$ is the socialization effort for parents related to education (e.g. participation in parental evenings, birthday parties, etc.). The more parents $i$ and $j$ participate in common parental activities, the more likely it is that they interact with each other. There is, quite naturally, congestion in socialization, as captured by $\rho(\mathbf{s})$.

The nature of this congestion is, of course an important part of the model, so some more discussion is in order. Taking the parenting example, interaction in parental evenings (of which there is a potentially unbounded number) happens in the form of conversations, which are always pairwise. The variable $s_{i}$ can be taken as the number of evenings a particular parent attends (meaning that $i$ can only devote a fixed amount of time to socialization activities). At the beginning of an evening, parents are paired at random for the duration of the event. Thus, the fraction of nights a parent $i$ is matched with parent $j$ is given by $\rho(\mathbf{s}) s_{j}$ and hence the total number of nights they interact is $\rho(\mathbf{s}) s_{j} s_{i}$. Congestion arises in this example because of the random matching technology. The higher is the number of parents, the more difficult is for a parent $i$ to be matched with a particular parent $j$.

The functional form in (1) and (2) can be tied back to simple properties of the link intensity $g_{i j}(\mathbf{s})$, as established below.

Lemma 1. Suppose that, for all $\mathbf{s} \neq \mathbf{0}$, the link intensity satisfies:

(A1) symmetry: $g_{i j}(\mathbf{s})=g_{j i}(\mathbf{s})$, for all $i, j$;

(A2) aggregate constant returns to scale: $\sum_{j=1}^{n} g_{i j}(\mathbf{s})=s_{i}$;

(A3) anonymous socialization: $g_{j i}(\mathbf{s}) / s_{j}=g_{k i}(\mathbf{s}) / s_{k}$, for all $i, j, k$;

then, the link intensity is given by (1) and (2).

Conditions (A2) and (A3) reflect the fact that $i$ controls his total number of contacts $s_{i}$, but the actual type composition of these contacts depends on others' socialization efforts. More precisely, each player devotes the same share of his total socialization effort to interacting with player $i$.

\subsection{Investment}

Each player $i$ makes a productive investment, $k_{i} \geqslant 0$. In our example, $k_{i}$ expresses parent's productive effort with the child (like e.g. doing homework and driving the child to different activities). The choices of $s_{i}$ and $k_{i}$ are simultaneous. Let $\mathbf{k}=\left(k_{1}, \ldots, k_{n}\right)$ be a profile of investments. These individual investments yield both a private and a synergistic return.

The private returns to player $i$ only depend on his own investment level $k_{i}$ and his idiosyncratic traits, summarized by $b_{\tau(i)} \geqslant 0$. We adopt a simple quadratic expression $b_{\tau(i)} k_{i}-c k_{i}^{2} / 2$.

The synergistic return depends on both $\mathbf{s}$ and $\mathbf{k}$. We consider multiplicative synergies $k_{i} k_{j}$ that are additively separable across pairs of players. For each pair of players $i, j$, these cross effects are scaled by a factor that reflects the intensity of the interaction between the two players. More precisely, we assume that:

$$
\frac{\partial^{2} u_{i}(\mathbf{s}, \mathbf{k})}{\partial k_{i} \partial k_{j}}=a g_{i j}(\mathbf{s}), \quad \text { for all } i \neq j,
$$

where $a \geqslant 0$ corresponds to the level of synergistic returns.

Notice that the symmetry (A1) in Lemma 1 is tantamount to payoffs being twice continuously differentiable in the productive investments $\mathbf{k}$. 


\subsection{Payoffs}

Let $c>0$. Player $i$ 's utility is equal to:

$$
u_{i}(\mathbf{s}, \mathbf{k})=b_{\tau(i)} k_{i}+a \sum_{j=1, j \neq i}^{n} g_{i j}(\mathbf{s}) k_{j} k_{i}-\frac{1}{2} c k_{i}^{2}-\frac{1}{2} s_{i}^{2} .
$$

Payoffs are a linear-quadratic function of $k_{i}$ s with non-negative cross effects (3) reflecting strategic complementarities in productive investments. The size $a g_{i j}(\mathbf{s}) \geqslant 0$ of these complementarities depends on the profile of socialization efforts, and varies across different pairs of players. For all $\mathbf{x} \in \mathbb{R}_{+}^{t}$, define $\overline{\mathbf{x}}=\sum_{\tau=1}^{t} x_{\tau} / t$ and $\overline{\mathbf{x}^{2}}=\sum_{\tau=1}^{t} x_{\tau}^{2} / t$. Then, other patterns of complementarities are given by:

$$
\begin{aligned}
& \frac{\partial^{2} u_{i}(\mathbf{s}, \mathbf{k})}{\partial s_{i} \partial s_{j}}=\frac{a k_{i} k_{j}}{n \overline{\mathbf{s}}}-\frac{a s_{i} k_{i} k_{j}}{(n \overline{\mathbf{s}})^{2}}+\sum_{l=1, l \neq i}^{n}\left(-\frac{a s_{l} k_{i} k_{l}}{(n \overline{\mathbf{s}})^{2}}+\frac{a g_{i l}(\mathbf{s}) k_{i} k_{l}}{(n \overline{\mathbf{s}})^{2}}\right)=\left(1-\frac{s_{i}}{n \overline{\mathbf{s}}}\right) \frac{a k_{i}}{n \overline{\mathbf{s}}}\left(k_{j}-\sum_{l=1, l \neq i}^{n} k_{l} \frac{s_{l}}{n \overline{\mathbf{s}}}\right), \\
& \frac{\partial^{2} u_{i}(\mathbf{s}, \mathbf{k})}{\partial s_{i} \partial k_{j}}=\frac{a s_{j} k_{i}}{n \overline{\mathbf{s}}}-\frac{a g_{i j}(\mathbf{s}) k_{i}}{n \overline{\mathbf{s}}}=\frac{a k_{i} s_{i}}{n \overline{\mathbf{s}}}\left(1-\frac{s_{i}}{n \overline{\mathbf{s}}}\right)>0 \\
& \frac{\partial^{2} u_{i}(\mathbf{s}, \mathbf{k})}{\partial k_{i} \partial s_{j}}=\frac{a s_{i} k_{j}}{n \overline{\mathbf{s}}}-\frac{a g_{i j}(\mathbf{s}) k_{j}}{n \overline{\mathbf{s}}}=\frac{a s_{i} k_{j}}{n \overline{\mathbf{s}}}\left(1-\frac{s_{j}}{n \overline{\mathbf{s}}}\right)>0 .
\end{aligned}
$$

Also let us note that

$$
\frac{\partial u_{i}}{\partial s_{j}}(\mathbf{s}, \mathbf{k})=\frac{a s_{i} k_{i} k_{j}}{n \overline{\mathbf{s}}}-\sum_{l=1}^{n} \frac{a s_{i} s_{l} k_{i} k_{l}}{(n \overline{\mathbf{s}})^{2}}=\frac{a s_{i} k_{i}}{n \overline{\mathbf{s}}}\left(k_{j}-\sum_{l=1}^{n} k_{l} \frac{s_{l}}{n \overline{\mathbf{s}}}\right) .
$$

Most of the interactions are positive with the exception of whether the socialization of a particular person $j$ creates positive or negative externalities on $i$ and whether the marginal benefit of socialization of $i$ increases or decreases on the socialization effort of $j$. In both cases, the effect on $i$ depends on whether person $j$ is making higher or lower effort $k_{j}$ than an average of the others (weighted by their own relative socialization effort).

Let us explain this utility function in the light of our education example. The term $b_{\tau(i)} k_{i}+a \sum_{j=1, j \neq i}^{n} g_{i j}(\mathbf{s}) k_{j} k_{i}$ is the outcome of the child (future wage or current grade) while $-\frac{1}{2} c k_{i}^{2}-\frac{1}{2} s_{i}^{2}$ is the cost of both efforts for parent $i$. In other words, the educational outcome of a child depends on $b_{\tau(i)}$, which represents either parental education or child's own talent (for example, I.Q.), $k_{i}$, the productive effort his parent is exerting in educating him, and $s_{i}$, the socialization effort his parent provides when participating in parental activities. The latter will help parent $i$ to be more productive (because of local complementarities) in educating his child. Indeed, the more a parent participates in different parental activities, the higher is the probability that he interacts with other parents and the more this parent will learn from them.

This general way of modeling the utility function in the context of education is similar to others in the literature (see, e.g. Bisin and Verdier, 2000; Patacchini and Zenou, 2010). The main novelty here is the way in which we model socialization through $g_{i j}(\mathbf{s})$ and its interaction with productive efforts $k_{i}$ and $k_{j}$.

\section{Equilibrium analysis and welfare}

\subsection{Equilibrium analysis}

We solve for the interior Nash equilibria in pure strategies $\left(\mathbf{s}^{*}, \mathbf{k}^{*}\right)=\left(s_{1}^{*}, \ldots, s_{n}^{*}, k_{1}^{*}, \ldots, k_{n}^{*}\right)$ of the $m$-replica game with heterogeneous types $\mathbf{b}=\left(b_{1}, \ldots, b_{t}\right)$, and for $m$ large enough. ${ }^{8}$

Under some conditions that we provide, there are exactly three such equilibria.

In one (partially corner) equilibrium, the level of socialization effort is null for all players. Indeed, non-reciprocated unilateral socialization efforts do not yield any interaction supportive of synergies. The two other equilibria are interior.

We first identify the (partially) corner equilibrium of the game.

Lemma 2. For all $m$-replica game, $\left(s_{i}^{*}, k_{i}^{*}\right)=\left(0, b_{\tau(i)} / c\right)$ for all $i=1, \ldots, m t$ is a pure strategy Nash equilibrium with corresponding equilibrium payoffs $b_{\tau(i)}^{2} / 2 c$.

\footnotetext{
8 Instead of having a m-replica game with heterogeneous types and large population, we could have instead considered a model with a continuum of agents and types. In that case, we would have obtained exactly the same results, except for the fact that $a(\mathbf{b})$ (defined below in Eq. (5)) would have been an integral rather than a sum. However, adopting a model with a continuum of players would make it more complicated for readers to link our approach to the one used in the network literature. That would obscure an important point of the paper linking network formation to network use.
} 
The intuition runs as follows. If nobody exerts any socialization effort, then it is optimal for other players not to provide a positive socialization effort and thus $s_{i}^{*}=0$. This is a Nash equilibrium. This equilibrium is a strict equilibrium, thus it cannot be discarded on the basis of standard refinements.

Define:

$$
a(\mathbf{b})=a \frac{\sum_{\tau=1}^{t} b_{\tau}^{2}}{\sum_{\tau=1}^{t} b_{\tau}} .
$$

Holding the average type $\sum_{\tau=1}^{t} b_{\tau} / t$ constant, the parameter $a(\mathbf{b})$ increases with the heterogeneity in types. More generally, $a(\mathbf{b})$ increases with the ratio $\sum_{\tau=1}^{t} b_{\tau}^{2} / \sum_{\tau=1}^{t} b_{\tau}$, which many authors refer to as the second-order average type (e.g., Vega-Redondo, 2007). When types are all homogeneous, that is, $b_{1}=\cdots=b_{t}=b$, we have $a(\mathbf{b})=a b$.

Theorem 1. Suppose that $2(c / 3)^{3 / 2}>a(\mathbf{b})>0$. Then, there exists an $m^{*}$ such that for all $m$-replica games with $m \geqslant m^{*}$, there are exactly two interior pure strategy Nash equilibria. These pure strategy Nash equilibria are such that, for all players $i$ of type $\tau$, the strategies $\left(s_{i}, k_{i}\right)$ converge to $\left(s_{\tau(i)}^{*}, k_{\tau(i)}^{*}\right)$ as $m$ goes to infinity, where $s_{\tau(i)}^{*}=b_{\tau(i)} s, k_{\tau(i)}^{*}=b_{\tau(i)} k$, and $(s, k)$ are positive solutions to:

$$
\left\{\begin{array}{l}
s=a(\mathbf{b}) k^{2} \\
k[c-a(\mathbf{b}) s]=1
\end{array}\right.
$$

In words, when $a(\mathbf{b})$ is small enough compared to the infra-marginal cost for a productive investment, the system of two equations (6) with two unknowns has exactly two positive solutions. As $m$ gets large, each such solution gets arbitrarily close to a pure strategy Nash equilibrium of the corresponding $m$-replica game. We get two approximate Nash equilibria. Besides, as $m$ gets large, every pure strategy Nash equilibrium gets arbitrarily close to a solution of (6).

A complete proof is derived in Appendix A. But we now provide a sketch of the proof in order to get a basic understanding of the result. Let $\mathbf{G}(\mathbf{s})=\left[g_{i j}(\mathbf{s})\right]_{i, j \in N}$ and $\operatorname{diag}(\mathbf{G}(\mathbf{s}))$ be a matrix with diagonal terms $g_{i i}(\mathbf{s})$ and zero off-diagonal terms. Then, the first-order condition with respect to $\mathbf{k}$ is

$$
[c \mathbf{I}-a \mathbf{G}(\mathbf{s})] \cdot \mathbf{k}+a \operatorname{diag}(\mathbf{G}(\mathbf{s})) \cdot \mathbf{k}=\mathbf{b} .
$$

Observe that the term with $\operatorname{diag}(\mathbf{G}(\mathbf{s}))$ appears in (7) because we allow for self-loops (when $i=j$ ) in $g_{i j}(\mathbf{s})$. The matrix $[c \mathbf{I}-a \mathbf{G}(\mathbf{s})]$ can be shown (Debreu and Herstein, 1953) to be invertible and has a particularly simple structure so that (7) becomes (see Lemma 3 in Appendix A):

$$
c \mathbf{k}+a\left[\mathbf{I}+\lambda_{a / c}(\mathbf{s}) \mathbf{G}(\mathbf{s})\right] \cdot \operatorname{diag}(\mathbf{G}(\mathbf{s})) \cdot \mathbf{k}=\left[\mathbf{I}+\lambda_{a / c}(\mathbf{s}) \mathbf{G}(\mathbf{s})\right] \cdot \mathbf{b},
$$

where $\lambda_{a / c}(\mathbf{s})=\frac{a}{c} \overline{\mathbf{c}} /\left(\overline{\mathbf{s}}-\frac{a}{c} \overline{\mathbf{s}^{2}}\right)$. The first-order conditions for $\mathbf{s}$ are:

$$
s_{i}=a k_{i} \frac{\mathbf{s} \cdot \mathbf{k}}{n \overline{\mathbf{s}}}-a s_{i} k_{i} \frac{\mathbf{s} \cdot \mathbf{k}}{(n \overline{\mathbf{s}})^{2}}-a \frac{s_{i} k_{i}^{2}}{n \overline{\mathbf{s}}}+a \frac{s_{i}^{2} k_{i}^{2}}{(n \overline{\mathbf{s}})^{2}}, \quad i=1, \ldots, n,
$$

where $\mathbf{s} \cdot \mathbf{k}=\sum_{j=1}^{n} s_{j} k_{j}$. Since $c>a \overline{\mathbf{s}^{2}} / \overline{\mathbf{s}}$ (which is necessary for $\lambda_{a / c}(\mathbf{s})$ to be well defined), $s_{i}$ is bounded in the limit for large $n$. Since $\mathbf{s}$ is bounded, one can see from (7) that $\mathbf{k}$ must also be bounded. And hence as the number of individuals for all types go to infinity we have that in the limit (8) becomes:

$$
s_{i}=a k_{i} \frac{\mathbf{s} \cdot \mathbf{k}}{n \overline{\mathbf{s}}}, \quad \text { for all } i=1, \ldots, n,
$$

since the terms $\mathbf{s} \cdot \mathbf{k} /(n \overline{\mathbf{s}})^{2}, s_{i} k_{i}^{2} / n \overline{\mathbf{s}}$ and $s_{i}^{2} k_{i}^{2} /(n \overline{\mathbf{s}})^{2}$ all go to zero when $n$ goes to infinity. Moreover,

$$
c \mathbf{k}=\left[\mathbf{I}+\lambda_{a / c}(\mathbf{s}) \mathbf{G}(\mathbf{s})\right] \cdot \mathbf{b},
$$

since $g_{i i}(\mathbf{s})=s_{i}^{2} / \sum_{j=1}^{n} s_{j}$ and hence $\operatorname{diag}(\mathbf{G}(\mathbf{s}))$ vanishes in the limit as $n$ becomes large.

From (9) notice that $s_{i} / k_{i}$ is a constant for all types so that one can write $s_{i}=\theta_{i} s$ and $k_{i}=\theta_{i} k$, for all $i$ and for some $k$, $s$ and $\theta_{i}$. Then, (9) can be rewritten as:

$$
s=a k^{2} \frac{\overline{\theta^{2}}}{\overline{\boldsymbol{\theta}}} \text {. }
$$

Note also that $\lambda_{a / c}(\mathbf{s})=a \overline{\boldsymbol{\theta}} /\left(c \overline{\boldsymbol{\theta}}-a s \overline{\boldsymbol{\theta}^{2}}\right)$ and thus (10) can be written as:

$$
c \theta_{i} k=b_{i}+\frac{a s}{n} \frac{\theta_{i}}{c \overline{\boldsymbol{\theta}}-a s \overline{\boldsymbol{\theta}^{2}}} \sum_{j=1}^{n} \theta_{j} b_{j} .
$$


Eq. (12) implies that for any $i, j$ we must have $\theta_{i} / \theta_{j}=b_{i} / b_{j}$ and then, without loss of generality, we can write $\theta_{i}=b_{i}{ }^{9}$ Then, (12) is equivalent to:

$$
k=\frac{1}{c-a(\mathbf{b}) s}
$$

and (11) is equivalent to:

$$
s=a k^{2} \frac{\overline{\mathbf{b}^{2}}}{\overline{\mathbf{b}}},
$$

which lead to (6).

The second-order conditions are satisfied at both equilibria. Since the reasons for why this is true are related to those for which (and why) equilibria are stable we briefly postpone the discussion, since we now study the stability of the equilibria described in Lemma 2 and Theorem 1.

Proposition 1. For $m$ sufficiently large, the two interior equilibria are stable while the equilibrium with $\left(s_{i}^{*}, k_{i}^{*}\right)=\left(0, b_{\tau(i)} / c\right)$ for all $i=1, \ldots, m t$ is not stable.

First, suppose we start with an equilibrium where everybody provides zero socialization effort, i.e. $\left(s_{i}^{*}, k_{i}^{*}\right)=\left(0, b_{\tau(i)} / c\right)$ for all $i=1, \ldots, m t$. If in this situation some player $j$ provides a positive socialization effort, then the marginal revenue of $s_{i}$, for player $j \neq i$, increases by a discrete amount (which is $a k_{i} k_{j}$ ) even for a small value of $s_{j}$, while the marginal cost of $s_{i}$ is small if $s_{i}$ is small. This result does not rely on the linear quadratic structure but on the fact that benefits and costs have different orders of power. From an economic viewpoint, any socialization effort, however small, leads to a social interaction that generates synergy payoffs when at least one additional agent socializes as well, which induces every other agent to socialize further.

This does not happen for the two interior equilibria described in Theorem 1. We consider a gradient dynamical system (see e.g. Corchón and Mas-Colell, 1996) for the dynamics of $(\mathbf{s}(t), \mathbf{k}(t))$ so that a variable is adjusted in a direction where payoffs improve (agents may only be imperfectly aware of their environment and hence adjust their strategy in a direction where they know their utility grows). Hence,

$$
\frac{\partial s_{i}(t)}{\partial t}=\frac{\partial u_{i}(\mathbf{s}(t), \mathbf{k}(t))}{\partial s_{i}(t)}, \quad \frac{\partial k_{i}(t)}{\partial t}=\frac{\partial u_{i}(\mathbf{s}(t), \mathbf{k}(t))}{\partial k_{i}(t)} .
$$

By linearizing around the equilibrium, we obtain:

$$
\begin{aligned}
\frac{\partial s_{i}(t)}{\partial t} & =\sum_{j=1}^{n^{h}} \frac{\partial^{2} u_{i}}{\partial s_{i} \partial s_{j}}\left(\mathbf{s}^{*}, \mathbf{k}^{*}\right)\left(s_{j}(t)-s_{j}^{*}\right)+\sum_{j=1}^{n^{h}} \frac{\partial^{2} u_{i}}{\partial s_{i} \partial k_{j}}\left(\mathbf{s}^{*}, \mathbf{k}^{*}\right)\left(k_{j}(t)-k_{j}^{*}\right), \\
\frac{\partial k_{i}(t)}{\partial t} & =\sum_{j=1}^{n^{h}} \frac{\partial^{2} u_{i}}{\partial k_{i} \partial k_{j}}\left(\mathbf{s}^{*}, \mathbf{k}^{*}\right)\left(k_{j}(t)-k_{j}^{*}\right)+\sum_{j=1}^{n^{h}} \frac{\partial^{2} u_{i}}{\partial k_{i} \partial s_{j}}\left(\mathbf{s}^{*}, \mathbf{k}^{*}\right)\left(s_{j}(t)-s_{j}^{*}\right) .
\end{aligned}
$$

In the limit for large $n$ and for $i \neq j$, we obtain:

$$
\frac{\partial^{2} u_{i}}{\partial s_{i} \partial s_{j}}\left(\mathbf{s}^{h *}, \mathbf{k}^{h *}\right)=\frac{\partial^{2} u_{i}}{\partial s_{i} \partial k_{j}}\left(\mathbf{s}^{h *}, \mathbf{k}^{h *}\right)=\frac{\partial^{2} u_{i}}{\partial k_{i} \partial s_{j}}\left(\mathbf{s}^{h *}, \mathbf{k}^{h *}\right)=\frac{\partial^{2} u_{i}}{\partial k_{i} \partial k_{j}}\left(\mathbf{s}^{h *}, \mathbf{k}^{h *}\right)=0
$$

and

$$
\frac{\partial^{2} u_{i}}{\partial s_{i}^{2}}\left(\mathbf{s}^{*}, \mathbf{k}^{*}\right)=-1, \quad \frac{\partial^{2} u_{i}}{\partial s_{i} \partial k_{i}}\left(\mathbf{s}^{*}, \mathbf{k}^{*}\right)=a(\mathbf{b}) k, \quad \frac{\partial^{2} u_{i}}{\partial k_{i}^{2}}\left(\mathbf{s}^{*}, \mathbf{k}^{*}\right)=-c,
$$

so that stability is determined in the limit by the eigenvalue of the following matrix $\boldsymbol{\Pi}^{h}$ :

$$
\boldsymbol{\Pi}^{h}=\left[\begin{array}{cc}
\mathbf{A}, & \mathbf{B} \\
\mathbf{B}, & c \mathbf{A}
\end{array}\right] \text { where } \mathbf{A}=\left[\begin{array}{ccc}
-1 & \ldots & 0 \\
\ldots & \ldots & \ldots \\
0 & \ldots & -1
\end{array}\right] \text { and } \mathbf{B}=\left[\begin{array}{ccc}
a(\mathbf{b}) k & \ldots & 0 \\
\ldots & \ldots & \ldots \\
0 & \ldots & a(\mathbf{b}) k
\end{array}\right],
$$

\footnotetext{
9 This also allows us to see that we are not focusing on the subset of symmetric equilibria, that is, on equilibria where individuals of the same type choose the same action. To see this, notice that we can arbitrarily split a type $t \in T$ into two groups: $t_{1}$ and $t_{2}$. But if both groups keep the same $b_{t}$, since the equilibrium action only depends on $b_{t}$, their actions will not change. So the commonality of actions is a requirement of all limits of equilibria, not a consequence of us focusing on symmetric equilibria.
} 
Table 1

Simulations with $a=2, c=1, t=1$ and $b=0.1$. $^{\text {a }}$

\begin{tabular}{|c|c|c|c|c|c|c|c|c|}
\hline$m$ & 2 & 5 & 10 & 20 & 50 & 100 & 500 & $\infty$ \\
\hline \multicolumn{9}{|c|}{ Low equilibrium } \\
\hline$k^{*}$ & 1898 & 1195 & 1101 & 1065 & 1049 & 1046 & 1046 & 1046 \\
\hline$s^{*}$ & 2366 & 815 & 458 & 303 & 234 & 222 & 218 & 219 \\
\hline \multicolumn{9}{|c|}{ High equilibrium } \\
\hline$k^{*}$ & 3346 & 4643 & 4591 & 4508 & 4444 & 4420 & 4400 & 4394 \\
\hline$s^{*}$ & 3506 & 3923 & 3911 & 3891 & 3875 & 3869 & 3864 & 3862 \\
\hline
\end{tabular}

a Numbers in the table must be multiplied by $10^{-3}$ to obtain the real equilibrium values.

where $\mathbf{A}, \mathbf{B}$ and $c \mathbf{A}$ are all diagonal matrices with $-1, a(\mathbf{b}) k$ and $-c$ in their diagonal, respectively. It is now easy to demonstrate that the equations for the eigenvalues have a non-trivial solution if and only if $(\lambda+1)(\lambda+c)-[a(\mathbf{b}) k]^{2}=0$. This second-order equation in $\lambda$ has a discriminant equal to $(c-1)^{2}+4[a(\mathbf{b}) k]^{2} \geqslant 0$, which always admits two real solutions, denoted $\lambda^{1}$ and $\lambda^{2}$. Besides

$$
\lambda^{1}+\lambda^{2}=-(1+c)<0,
$$

implying that at least one such solution is negative. Then, both are negative if and only if $\lambda^{1} \lambda^{2}=c-(a(\mathbf{b}) k)^{2}>0$, equivalent to $c-a(\mathbf{b}) s>0$, which is true when $2(c / 3)^{3 / 2}>a(\mathbf{b})$. This is the condition that guarantees that the second-order conditions are satisfied (see Theorem 1 ).

In words, the equations in (15) imply that there is no feedback from the changes in one individuals' strategies to any of the others' when close to either equilibria. Then, the local dynamics are entirely driven by one individual's strategy and thus local stability is the same as local maxima, which is why the condition for stability is the same as the condition that guarantees that the second-order conditions are satisfied.

Given that the partially corner equilibrium is unstable for high enough replications, we concentrate on the interior equilibria. Table 1 shows the discrepancy between equilibrium and approximated equilibrium actions for various population sizes when the population is homogeneous with common trait $b$. The last column corresponds to the approximated equilibrium actions; the other columns give the exact Nash equilibrium actions as $m$ varies.

For an homogeneous population with common trait $b$, one can verify that the exact equilibrium equations are:

$$
\left\{\begin{array}{l}
s=a b k^{2}\left(1-\frac{1}{m}\right)^{2}, \\
k\left[c-a b s\left(1-\frac{1}{m}\right)\right]=1 .
\end{array}\right.
$$

Comparing with (6), it can be checked that the approximation error is of the order of $m^{-3 / 2}$. In particular, when $m=100$, the approximation error is of the order $10^{-3}$.

The approximated equilibria $\left(\mathbf{s}^{*}, \mathbf{k}^{*}\right)$ characterized in Theorem 1 display three important features.

First, the level of socialization per unit of productive investment is the same for all players, that is, $s_{i}^{*} / k_{i}^{*}=s_{j}^{*} / k_{j}^{*}$, for all $i, j$. Given our quadratic cost structure, this is equivalent to having a marginal rate of substitution of socialization versus investment for gross benefits uniform across all players. An approximate equilibrium is thus fully characterized by specifying the value of this ratio together with the population profile of productive investments.

Second, differences in productive investments reflect differences in idiosyncratic traits. More precisely, $k_{i}^{*} / k_{j}^{*}=b_{\tau(i)} / b_{\tau(j)}$, for all $i, j$. Indeed, absent of any synergy payoffs, private returns are maximal when productive investment is equal to $b_{\tau(i)} / c$, and the ratio of these maximal investments is then also equal to $b_{\tau(i)} / b_{\tau(j)}$. At an approximate equilibrium, the relative value of productive investments thus remains unchanged with and without synergies. The presence of synergies only affects the absolute value of those investments.

Third, in the presence of synergies, productive investments are all scaled up (compared to the case without synergies) by a synergistic multiplier. ${ }^{10}$ This multiplier, which is homogeneous across all players, is a decreasing function of the infra-marginal productive investment cost $c$, and an increasing function of the second-order average type $a(\mathbf{b})$. Beyond this dependence on exogenous payoff parameters, the synergistic multiplier also depends on the endogenous baseline equilibrium socialization effort $s^{*}$.

In substance, individual traits enter multiplicatively into own actions at approximate equilibria. Also, the population-wide heterogeneity collapses into a single index, $a(\mathbf{b})$. Compared with the case without synergies, productive investments are all scaled up by a common synergistic multiplier that depends on this index. Finally, the ratio of socialization to productive effort is uniform across all agents.

\footnotetext{
10 The multiplier is, precisely, $1 /\left(1-a(\mathbf{b}) s^{*} / c\right)$. This is obtained by rewriting the second equation of $(6)$ as $k_{i}^{*}=b_{\tau(i)} k^{*}=b_{\tau(i)} /\left(c-a(\mathbf{b}) s^{*}\right)$, and by dividing the right-hand term by the optimal productive investment level in the absence of synergies, $b_{\tau(i)} / c$.
} 


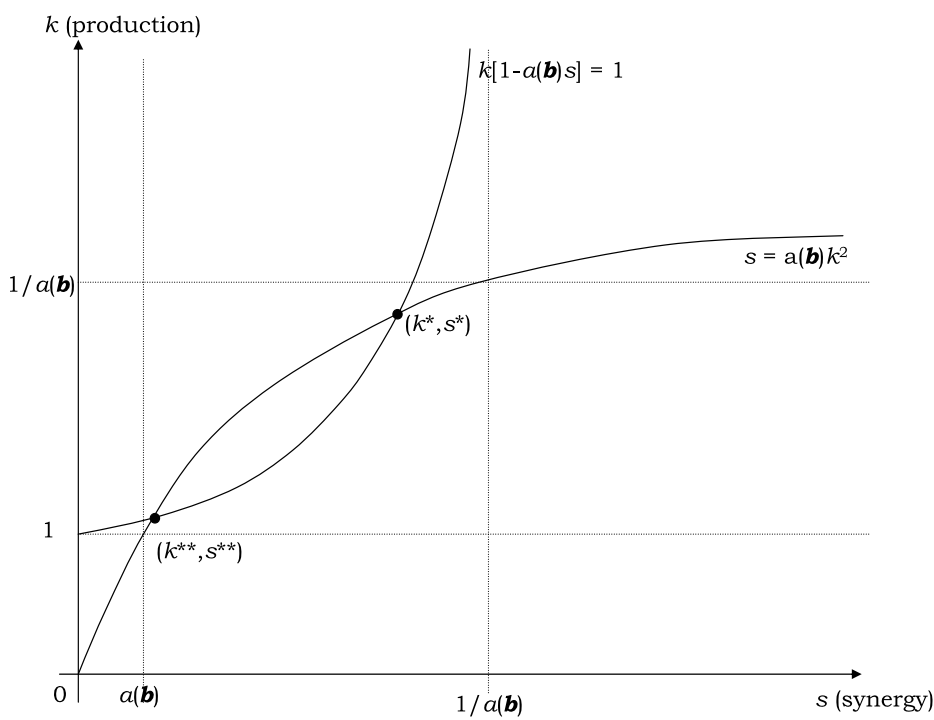

Fig. 1.

These properties of the equilibrium actions have implications for the type composition of the social interaction circle of each player. Recall that $s_{i}^{*}$ gives the total interaction intensity, or size, of the social circle of agent $i$. We deduce from the above discussion that social circles across players of different types $\tau$ and $\tau^{\prime}$ vary in fixed proportions $b_{\tau} / b_{\tau^{\prime}}$. Beyond this size effect, the inner composition of social circles is the same for all agents. More precisely, every agent $i$ devotes an identical fraction $b_{\tau^{\prime}} / \sum_{\tau \in T} b_{\tau}$ of his total socialization intensity to interacting with type $\tau^{\prime}$ agents, and this fraction is independent of his own type.

Fig. 1 plots Eqs. (6).

From the graph, it is clear that the system (6) need not always have a non-negative solution. The upper bound on $a(\mathbf{b})$ in Theorem 1 is a necessary and sufficient condition for the two graphs to cross in the positive orthant of the space $(s, k)$. When $a(\mathbf{b})$ is too large, the synergistic multiplier operates too intensively and there is no intersection. More precisely, holding $s$ fixed, the level of productive investments escalates without bound. In turn, this triggers and unbounded increase in socialization effort, and both effects positively feed back into each other.

Remark 1. When $0<a(\mathbf{b})<2(c / 3)^{3 / 2}$, the system of Eqs. (6) has two different non-negative solutions. When $a(\mathbf{b})=$ $2(c / 3)^{3 / 2}$, there is a unique non-negative solution $(s, k)=(3 / c, \sqrt{3 / c}) / 2$. When $a(\mathbf{b})>2(c / 3)^{3 / 2}$, there is no non-negative solution.

One slightly artificial feature of the model is the fact that the effort variables are unbounded. This creates existence problems and generates the need for the assumption $a(\mathbf{b})<2(c / 3)^{3 / 2}$. In addition, this generates a failure of upperhemicontinuity in the equilibrium correspondence as the high-action equilibrium diverges to infinity as $a(\mathbf{b})$ goes to zero. A simple way to deal with this problem is to assume that the effort of each individual is bounded. That is, $s_{i}+k_{i} \leqslant T$. This is natural when one interprets this effort as an activity that consumes resources. It is relatively easy to characterize the equilibria in this case. In particular, the Pareto-superior equilibrium (see below) disappears for $a(\mathbf{b}) T$ low enough. A bounded strategy space can also introduce upper corner equilibria which may be stable.

\subsection{Welfare}

Given an approximate equilibrium $\left(\mathbf{s}^{*}, \mathbf{k}^{*}\right)$, we denote by $\mathbf{u}\left(\mathbf{s}^{*}, \mathbf{k}^{*}\right)=\left(u_{1}\left(\mathbf{s}^{*}, \mathbf{k}^{*}\right), \ldots, u_{m}\left(\mathbf{s}^{*}, \mathbf{k}^{*}\right)\right)$ the corresponding equilibrium payoffs. Denote also by and $\left(\mathbf{s}^{E}, \mathbf{k}^{E}\right)$ the (approximate) efficient outcome, i.e., the one (almost) maximizing the sum of payoffs for all players in a large $m$-replica game.

The next result compares equilibrium actions and payoffs across the two approximate equilibria characterized in Theorem $1 .{ }^{11}$

\footnotetext{
11 Notice that all the claims to follow are made on approximate equilibria rather than on the exact Nash equilibria of the game. Given the local continuity of the equilibrium correspondence around interior equilibria, the claims about approximate equilibria are generally portable to properties of Nash equilibria, although some qualifications may be sometimes required.
} 
Proposition 2. Assume $0<a(\mathbf{b})<2(c / 3)^{3 / 2}$ and let $\left(\mathbf{s}^{*}, \mathbf{k}^{*}\right)$ and $\left(\mathbf{s}^{* *}, \mathbf{k}^{* *}\right)$ be the two different approximate equilibria of an $m$-replica game. Then, without loss of generality, $\left(\mathbf{s}^{*}, \mathbf{k}^{*}\right) \geqslant\left(\mathbf{s}^{E}, \mathbf{k}^{E}\right) \geqslant\left(\mathbf{s}^{* *}, \mathbf{k}^{* *}\right)$ and $\mathbf{u}\left(\mathbf{s}^{E}, \mathbf{k}^{E}\right) \geqslant \mathbf{u}\left(\mathbf{s}^{*}, \mathbf{k}^{*}\right) \geqslant \mathbf{u}\left(\mathbf{s}^{* *}, \mathbf{k}^{* *}\right)$, where $\geqslant$ is the component-wise ordering.

In words, the equilibrium actions are ranked component-wisely and the equilibrium payoffs are Pareto-ranked accordingly. From now on, we refer to the Pareto-superior and to the Pareto-inferior approximate equilibrium as the high and the low equilibrium, respectively. The socially efficient outcome lies in between the two equilibria, so that we can effectively talk about a too-high and a too-low equilibrium. To get an intuition for why the efficient outcome lies in between the equilibria, note that the first-order conditions for efficiency in the limit economy look as follows:

$$
\left\{\begin{array}{l}
s=2 a(\mathbf{b}) k^{2}, \\
k[c-2 a(\mathbf{b}) s]=1 .
\end{array}\right.
$$

These conditions are as in (6) but with $2 a(\mathbf{b})$ replacing $a(\mathbf{b})$, as a result of the externalities caused by agents' actions of others they do not take into account when maximizing their own welfare. But Proposition 3 shows that an increase in $a(\mathbf{b})$ leads to new equilibria where the new high equilibrium is below the earlier one, and the low equilibrium is above the previous one. Hence, the solutions to (16) will lie in between equilibrium solution. Of those solutions the welfare maximizer will be the higher one for reasons that are analogous to why the high equilibrium has a higher welfare than the low equilibrium.

If we go back to our example on education, then this means that, compared to the socially efficient outcome, there is an equilibrium where parents put too much effort in socializing and educating their children and another one where they exert too little effort. This is due to local complementarities and positive synergies so that the too-high (too-low) equilibrium is reached because parents, who are obsessed with (not interested in) the education of their kids, meet other obsessed (noninterested) parents, which, in turn, make parents even more obsessed (uninterested), triggering even more (less) productive efforts on both side.

Denote by $\left(s^{*}, k^{*}\right)$ and $\left(s^{* *}, k^{* *}\right)$ the baseline socialization and productive efforts that solve (6), and that enter the calculation of the high and the low equilibrium, respectively. Then, it is already apparent from Fig. 1 that $s^{*} / k^{*} \geqslant s^{* *} / k^{* *}$. That is, the level of socialization per unit of productive investment is higher at the high equilibrium. At the high equilibrium, a high joint socialization effort creates tight links across players who then invest heavily in productive effort to build high cross synergies on this fertile ground. At the low equilibrium, low joint socialization efforts lead to a loose interaction pattern which hampers the scope for cross synergies, and thus the level of private investments. As a matter of fact, the synergistic multiplier is higher in the high equilibrium, and pulls up the level of production investments compared to the low equilibrium.

The equilibrium multiplicity identified in Theorem 1 reflects an inter-twinned coordination problem in the socialization process and in the production technology. These two coordination problems are rooted separately on the payoffs strategic complementarity both in socialization effort and in productive investment. The dependence of the cross returns in production on the population socialization profile, $\partial^{2} u_{i}(\mathbf{s}, \mathbf{k}) / \partial k_{i} \partial k_{j}=a g_{i j}(\mathbf{s})$ for $i \neq j$, relates these two coordination problems with each other, as reflected by the endogenous dependence of the synergistic multiplier on the baseline socialization effort.

To sum up, we have characterized the set of equilibria for the model when a sufficiently large number of agents participates in the game. We have shown that there are two stable interior equilibria and one unstable corner equilibrium (without socialization). Existence and stability of interior equilibria are obtained when the level of cross synergies, as well as the heterogeneity in individual traits, are not too large. This amounts to bounding from above a compound index of both kinds of payoff parameters. In the absence of that bound, the complementarities inherent to the model would make equilibrium values of the variables to diverge. Finally, we have shown that the stable equilibria are Pareto-ranked, and that the socially efficient outcome lies in between the stable equilibria.

\section{Comparative statics analysis and empirical implications}

Recall that $a(\mathbf{b})$ is a compound index of the technological synergy parameter and of the second-order average type $\sum_{\tau=1}^{t} b_{\tau}^{2} / \sum_{\tau=1}^{t} b_{\tau}$, that measures the population heterogeneity in private returns to productive investment. This compound index directly enters in the equilibrium behavior (6) and, in particular, in the value of the synergistic multiplier.

Exogenous changes in the value of the technological parameter and/or in the group characteristics have an impact on socialization and investment that is channeled through the variations in $a(\mathbf{b})$ that follow these changes. The next result clarifies how socialization and investment react to changes in $a(\mathbf{b})$.

Proposition 3. Suppose that $a(\mathbf{b})$ increases. Then, in both approximate equilibria of the replica game, the percentage change in socialization effort is higher than that of productive investment for all agents. Besides, the baseline equilibrium actions that solve (6) both increase at the low equilibrium and decrease at the high equilibrium.

Recall that equilibrium actions are multiplicative in own traits, $\left(s_{i}^{*}, k_{i}^{*}\right)=b_{\tau(i)}\left(s^{*}, k^{*}\right)$, where $\left(s^{*}, k^{*}\right)$ are the baseline socialization and productive investments that solve (6). Proposition 3 implies that the socialization effort per unit of productive investment, $s_{i}^{*} / k_{i}^{*}$, decreases at the high equilibrium and increases at the low equilibrium when $a(\mathbf{b})$ goes up. It 


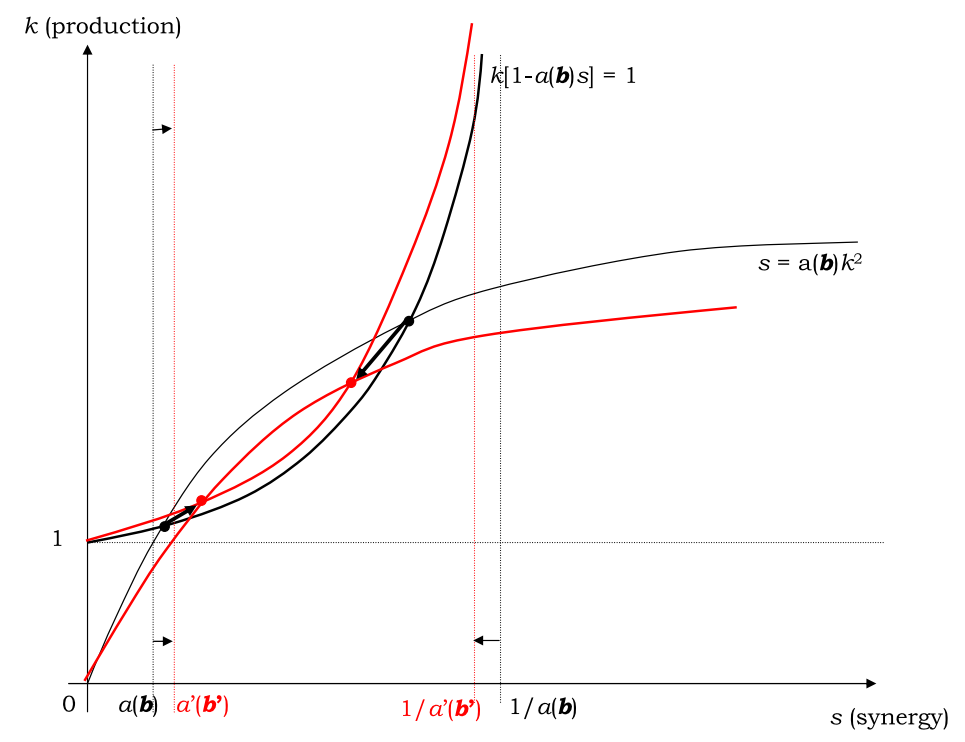

Fig. 2.

also states that the elasticity of socialization with respect to productive investment, that accounts for the relative variation of these two actions, is smaller than one at all equilibria.

At the high equilibrium, where socialization is overwhelming, agents substitute an increase in $a(\mathbf{b})$ by an endogenous decrease in baseline socialization $s^{*}$. As a result, the synergistic multiplier decreases and the baseline productive investment is pulled down. This decrease in production feeds back and further dampens the level of socialization. And so forth, until this chain of cross influences sets at a new equilibrium. It turns out that socialization is more responsive than productive investment.

At the low equilibrium, instead, where the interaction pattern is diffuse, agents complement an increase in $a(\mathbf{b})$ by an increase in socialization, which triggers an upwards jump in baseline productive investment. Again, socialization is here more responsive than productive investment.

The reason for this over-responsiveness of socialization can be better understood by remembering that elasticity is given by:

$$
\eta=\frac{k}{s} \frac{\frac{\partial s}{\partial a(\mathbf{b})}}{\frac{\partial k}{\partial a(\mathbf{b})}}=\frac{\frac{\partial \ln s}{\partial a(\mathbf{b})}}{\frac{\partial \ln k}{\partial a(\mathbf{b})}}
$$

but inspecting the first-order condition for socialization we see that

$$
\ln s=\ln a(\mathbf{b})+2 \ln k
$$

and hence

$$
\eta=\frac{1+2 \frac{\partial \ln k}{\partial a(\mathbf{b})}}{\frac{\partial \ln k}{\partial a(\mathbf{b})}}>2 .
$$

In words, the result arises because the marginal benefit of socialization is a function of $k^{2}$, whereas the marginal cost is a function of $s$. This result, as we discuss in more detail in Section 5, has interesting and novel empirical implications.

Fig. 2 illustrates how, at the low equilibrium, an increase in $a(\mathbf{b})$ leads to an increase in both socialization and investment. At the high equilibrium, instead, baseline socialization and investment decrease.

Changes in the compound index $a(\mathbf{b})$ can subsume various effects of very different nature. More precisely, this compound index responds positively to upwards shifts in the technological scale of synergistic returns $a$, to changes in the population that increase the second-order average type $\sum_{\tau=1}^{t} b_{\tau}^{2} / \sum_{\tau=1}^{t} b_{\tau}$, and to combination of both effects.

For instance, a mean-preserving spread in idiosyncratic traits leaves $\sum_{\tau=1}^{t} b_{\tau}$ constant while $\sum_{\tau=1}^{t} b_{\tau}^{2}$ grows. The ensuing upwards shift in the second-order average type increases $a(\mathbf{b})$.

Suppose also that $a$ and all the $b_{\tau}$ 's $a$ are all scaled up by a common factor. In particular, consider the following variation of payoffs (4), where $\lambda>0$ : 


$$
u_{i}(\mathbf{s}, \mathbf{k})=b_{\tau(i)} k_{i}+a \sum_{j=1, j \neq i}^{n} g_{i j}(\mathbf{s}) k_{j} k_{i}-\frac{\lambda}{2} c k_{i}^{2}-\frac{\lambda}{2} s_{i}^{2} .
$$

The game with payoffs (17) has the same equilibria than the game with original payoffs (4), where the types $b_{\tau}$ and the synergy scale parameter $a$, and thus the compound index $a(\mathbf{b})$, are all scaled homothetically by $1 / \lambda$.

Proposition 3 encompasses all those cases and many others, and pins down the relative variation of socialization and productive investment, and the absolute variation of baseline socialization and productive investment, for all these multifarious changes in parameters.

Notice, however, that equilibrium actions $\left(s_{i}^{*}, k_{i}^{*}\right)=b_{\tau(i)}\left(s^{*}, k^{*}\right)$ need not move in the same direction than baseline equilibrium actions $\left(s^{*}, k^{*}\right)$. This may be so at the low equilibrium when the changes in $a(\mathbf{b})$ result from variations in parameter values for which $b_{\tau(i)}$ moves in the opposite direction than $a(\mathbf{b})$, and at the high equilibrium if $b_{\tau(i)}$ moves in the same direction than $a(\mathbf{b})$.

The following result clarifies this point.

Corollary 1. An increase in $a(\mathbf{b})$ for which $b_{\tau}$ does not decrease (resp. does not increase) increases (resp. decreases) the socialization and investment efforts of all type $\tau$ players at the low equilibrium (resp. at the high equilibrium).

We now document the comparative statics of individual and aggregate equilibrium payoffs when $a(\mathbf{b})$ varies, with a particular emphasis on the effect of the group heterogeneity.

When $m$ gets large, approximate equilibrium payoffs corresponding to baseline efforts $\left(s^{*}, k^{*}\right)$ that solve (6) are given by the following expression:

$$
u_{i}^{*}=\frac{b_{\tau(i)}^{2}}{2 a(\mathbf{b})} \frac{s^{*}}{k^{*}}+o(1)=\frac{b_{\tau(i)}^{2}}{2} k^{*}+o(1), \quad \text { for all } i=1, \ldots, m t .
$$

Given the expression for equilibrium payoffs (18), the comparative statics of equilibrium actions established in Proposition 3 and Corollary 1 above have straight implications for the reaction of individual well being to changes in the compound index $a(\mathbf{b})$.

Proposition 4. An increase in $a(\mathbf{b})$ for which $b_{\tau}$ does not decrease (resp. does not increase) increases the low equilibrium payoffs (resp. decreases the high equilibrium payoffs) of all type $\tau$ players.

In particular, an increase in the synergy parameter $a$ induces a downwards shift of equilibrium payoffs at the high equilibrium, and an upwards shift at the low equilibrium. Indeed, scaling up the scope for synergies helps alleviating the coordination features of both equilibria. At the low equilibrium, characterized by an under-provision of production and socialization efforts, the interaction pattern is tightened and investments increment. At the high equilibrium, instead, where over-provision prevails, the social network is loosened and investments reduced.

Proposition 4 also documents the changes in individual welfare following a change in the population distribution of types. We explore some changes in the types profile $\mathbf{b}=\left(b_{1}, \ldots, b_{t}\right)$ that induce an increase in $a(\mathbf{b})$.

Consider two type profiles $\mathbf{b}^{\prime} \geqslant \mathbf{b}$, where $\geqslant$ is the component-wise ordering. The marginal private returns to productive investment of every agent are not smaller under $\mathbf{b}^{\prime}$ than under $\mathbf{b}$, and are strictly higher for at least one type when $\mathbf{b}^{\prime} \neq \mathbf{b}$. Even though $\mathbf{b}^{\prime}$ is obtained from $\mathbf{b}$ by increasing the type of some agents, it is not always true that the value of $a\left(\mathbf{b}^{\prime}\right)$ so obtained is higher than that of $a(\mathbf{b})$. The following results clarify this point.

Recall that $0<b_{1} \leqslant b_{2} \leqslant \cdots \leqslant b_{t}$.

Proposition 5. Let $\mathbf{b}=\left(b_{1}, \ldots, b_{t}\right)$ be a type profile such that $2 b_{1} \geqslant b_{t}$. Then for all $\tau \in T, \tau \neq \tau(i)$, $\partial u_{i}^{* *} / \partial b_{\tau}>0$ (i.e. the low equilibrium payoffs of every player increase with $\left.b_{\tau}\right)$, while $\partial u_{i}^{*} / \partial b_{\tau}<0$ (the high equilibrium payoffs decrease with $\left.b_{\tau}\right){ }^{12}$

Recall that, at equilibrium, all agents have the same distribution of types in their social circle, and interactions with a type $\tau$ represent a fraction $b_{\tau} / \sum_{\tau^{\prime} \in T} b_{\tau^{\prime}}$ of the agent's total socialization intensity. When $2 b_{1} \geqslant b_{t}$, the ratio of higher to lower type values is never higher than two. As a result, the type composition of agent's social circles are moderately uneven, and high types are not over-represented with respect to lower types.

With a moderately uneven type distribution, agents of different types contribute in not too disparate proportions to the creation of cross-synergies.

More precisely, the synergistic payoff to a bilateral interaction between players $i$ and $j$ is $a g_{i j}(\mathbf{s}) k_{i} k_{j}$. At equilibrium, we can rewrite this spillover as $a s_{i}^{*} s_{j}^{*} k_{i}^{*} k_{j}^{*} / \sum_{\tau \in T} b_{\tau} s^{*}$. Holding the average type constant, the two players contribute to (respectively, $s_{i}^{*} k_{i}^{*}$ and $s_{j}^{*} k_{j}^{*}$ ) the creation of the synergy payoffs. Relative contributions to the synergy are thus equal to $b_{\tau(i)}^{2} / b_{\tau(j)}^{2}$.

\footnotetext{
12 A weakening of the sufficient condition $2 b_{1} \geqslant b_{t}$ is to assume that $b_{\tau}^{2}+2 b_{\tau} \sum_{\tau^{\prime} \neq \tau} b_{\tau^{\prime}} \geqslant 2 \sum_{\tau^{\prime} \neq \tau} b_{\tau^{\prime}}^{2}$, for all $\tau \in T$.
} 
Two interacting players with identical type contribute symmetrically to the cross spillover they exert on each other, while two interacting players with different type contribute asymmetrically. When $2 b_{1} \geqslant b_{t}$, these asymmetric contributions are not too strikingly different, with values in $[1 / 4,4]$, and cross spillovers are moderately asymmetric.

With moderately asymmetric cross spillovers, any type value unilateral increase benefits all players at the low equilibrium who each increase the spillovers they exert to every other interacting partner.

Instead, when type values differ drastically with each other, players' contributions to the spillovers with players of a different type are highly asymmetric. An increase in the low type value can now decrease the welfare of high type players at the low equilibrium, as illustrated below.

Example 1. Consider two types $\mathbf{b}=\left(b_{1}, b_{2}\right)$ with $b_{1}<(\sqrt{2}-1) b_{2}$. Then, $a(\mathbf{b})$ is a strictly decreasing function of the low type $b_{1} \cdot{ }^{13}$ An increase in $b_{1}$ decreases the payoffs of the high type players at the low equilibrium.

When $b_{1}<(\sqrt{2}-1) b_{2}$, the fraction of type 2 players in every individual social circle is bounded from below by $1 / \sqrt{2}$, a lower bound of roughly $70.7 \%$ interactions with type 2 players. In particular, high type 2 players interact overwhelmingly with players of the same type. This in-breeding bias is accompanied by symmetric cross-spillovers across high type players to which they all contribute intensively. High type players also contribute asymmetrically to spillovers they exert on low type players. But the interactions sustaining these spillovers are rare, and they main concern is the creation of within-type symmetric spillovers.

Suppose now that the value of $b_{1}$ increases. The asymmetric high-low type interactions become more prevalent. A higher share of the socialization and production efforts of high type players is now beneficial to low type players, who partially free-ride on these spillovers to which they themselves don't contribute much. High type players then react to this increase in $b_{1}$ by narrowing down the size of their social circles at the low equilibrium and, more generally, by decreasing the generation of cross-spillovers that they now share symmetrically among themselves in a smaller proportion.

The previous results characterize the effect on individual payoffs of changes in type composition. Now following a mean preserving effect in types, the compound index $a(\mathbf{b})$ increases. This induces an upward shift of baseline actions at the low equilibrium. Individuals experiencing an increase in type will certainly experience an increase in payoffs, but the effect is ambiguous for agents experiencing a decrease in types. The next result shows, however, that the overall aggregate effect is positive. The argument is symmetric at the high equilibrium.

Proposition 6. Aggregate payoffs increase at the low equilibrium and decrease at the high equilibrium following a mean-preserving spread of the population type profile $\mathbf{b}=\left(b_{1}, \ldots, b_{t}\right) .{ }^{14}$

\section{Empirical implications in terms of education}

Let us now go back to our example in terms of education where each parent $i$ exerts two types of costly effort: productive effort $k_{i}$ with the child and socialization effort $s_{i}$ related to education. This interpretation of the model makes the empirical predictions more precise. We find that:

(i) Better educated parents exert more productive effort educating their kids than low-educated parents, i.e. $k_{i}$ and $b_{i}$ are positively related (Proposition 3). This fact is well documented. For example, for England, using the National Child Development Study, Patacchini and Zenou (2010) find that more educated parents put more effort in educating their children than less educated parents. More precisely, they find that around 70 percent of educated parents are highly interested in their child education (measured by the frequency they read to their child) while it is roughly 30 percent for less educated parents.

(ii) Better educated parents are more prone to socialize with other parents than low-educated parents, i.e. $s_{i}$ and $b_{i}$ are positively related (Proposition 3). There is also empirical evidence on this issue. Putnam (1995) documents that less educated people are less engaged in the life of their communities, in particular, group membership. Moreover, using data from the US General Social Survey (GSS) from 1972 through 1996 and from the DDB-Needham Life Style survey data from 1975 through 1997, Helliwell and Putnam (2007) investigate the effects of education on trust and social engagement, two key variables often used as measures of social capital. They find, in particular, that higher-educated parents are more likely to participate socially in school activities than low-educated parents. ${ }^{15}$

(iii) Our model has multiple equilibria. This feature (Theorem 1) can help to explain why, in different locales, children whose parents have similar characteristics (e.g. income, education level) or are similarly talented as other children (say, measured by I.Q.) end up having very different educational outcomes or different levels of parental educational efforts. Glaeser et al. (1996) make a similar observation about crime. Their argument runs as follows. If one compares different neighborhoods with the same fundamentals (in terms of unemployment rate, poverty and so on), one observes that crime

\footnotetext{
13 We have $a(\mathbf{b})=\left(b_{1}^{2}+b_{2}^{2}\right) /\left(b_{1}+b_{2}\right)$. The sign of $\partial a(\mathbf{b}) / \partial b_{1}$ is that of $b_{1}^{2}+2 b_{1} b_{2}-b_{2}^{2}$, which is negative when $\left(b_{1}+b_{2}\right)^{2}<2 b_{2}^{2}$, that is, $b_{1}<(\sqrt{2}-1) b_{2}$. 14 That leaves $\sum_{\tau=1}^{t} b_{\tau}$ constant but increases $\sum_{\tau=1}^{t} b_{\tau}^{2}$.

15 See also Dee (2004) and Milligan et al. (2004), which use instrumental variables to estimate the effects of own education. The results show that individuals with more education tend to be more engaged citizens.
} 
rates are very different. Social interactions, which are usually not observed by the econometrician, can explain these differences. The same argument can be used to test whether social interactions between parents with the same education levels can explain different educational outcomes of children.

(iv) Different levels of parental education affect (positively or negatively) proportionally more the socialization effort of those parents $s_{i}$ than their direct effort $k_{i}$ with children (Proposition 3). This is an interesting prediction that, to the best of our knowledge, has not been yet tested.

An important question is then how an exogenous change in the returns to production and socialization affect the relative production and socialization efforts at equilibrium. In turns out that, when the returns increase because either all $b_{i}$ increase or $a$ does, all equilibrium actions decrease at the Pareto-superior equilibrium, while they increase at the Paretoinferior equilibrium. In both cases, the percentage change in socialization effort is higher (in absolute value) than that of the productive effort.

To sum up, in this section we have shown that the comparative statics for the model are very tightly connected to the parameter $a(\mathbf{b})$. This allows us, for example, to relate the variations in equilibria with changes in the strength of incentives, or with relative population heterogeneity. We have also shown that socialization is more responsive than production to exogenous shocks in the parameters. Finally, we have explored some empirical implications of the model. Some are well established, and some are novel.

\section{Discussion}

In this paper, we provide a simple operational model of network formation with productive effort, synergies and population heterogeneity. An innovation of our study is that socializing is not equivalent to elaborating a nominal list of intended relationships, as in the literature on network formation. In other words, network formation does not result of an earmarked socialization process. This shortcut greatly improves the tractability of the model and allows us to perform a standard type of equilibrium analysis that equates marginal costs and benefits of both production and socialization. ${ }^{16}$ We obtain welfare predictions and clear-cut comparative statics. In substance, we identify a "too cold" and a "too hot" equilibrium. We show that socialization is more responsive than production to exogenous shocks in the parameters. We also demonstrate that our analysis is robust to different specifications generalizing the model.

As all models, ours relies on specific assumptions due to the functional form of payoffs given in Eq. (4). The three main characteristics of this functional form are:

(a) the linear-quadratic returns to productive investment,

(b) aggregate constant returns to scale in socialization effort (condition (A2) in Lemma 1),

(c) the generic socialization effort (condition (A3) in Lemma 1).

Combining the linear equilibrium equations for productive investment with conditions (A2) and (A3), Nash equilibrium conditions (both for productive investments and for socialization efforts) take a relatively manageable closed-form matrix expression. In turn, when the population gets large, and because we are able to control the population size effect in our matrix closed-form expression, approximate equilibrium conditions boil down to a simple system of Eqs. (6).

Our results (in terms of characterization of equilibria, multiple equilibria, welfare and comparative statics results) are robust to certain extensions of the model, ${ }^{17}$ that we discuss now.

(a) Linear-quadratic production payoffs give rise to linear equilibrium conditions for the levels of productive investment. Linear equilibrium conditions for productive investment play an important role in the analysis, as they allow to express existence and interiority of the productive investment decisions (for a given socialization profile s) as a function of the spectral radius of the matrix of link intensities $\mathbf{G}(\mathbf{s})=\left[g_{i j}(\mathbf{s})\right]$. However, it is important to stress that linear-quadratic payoffs are not a necessary condition for equilibrium equations to be linear. ${ }^{18}$ Indeed, equilibrium analysis boils down to solving a so-called linear complementarity problem for a relatively broad class of payoff function, beyond the particular linearquadratic specification. Ballester and Calvó-Armengol (forthcoming) provide examples of these payoff functions. Our analysis would carry over to any such environment. By considering non-quadratic cost structures, we can show that our analysis is robust to (at least some form of) non-linearities in these equilibrium conditions.

(b) Condition (A2) is mainly chosen for its operationally virtues. We can accommodate variations of this condition, and thus alternative expressions for the link intensities $g_{i j}(\mathbf{s})$ that allow for some aggregate scale effects in socialization. For example, instead of constant aggregate returns to scale, we can adopt non-constant returns to scale (i.e. increasing or decreasing) and show that most of our results still hold.

\footnotetext{
16 Because of its simplicity and flexibility, our model could be used to analyze different outcomes where social interactions matter. A recent paper by Galeotti and Merlino (2009) uses our network formation process to analyze social interactions and job search. They find interesting results that are empirical relevant. Golub and Livne (2010) also use a similar approach (i.e. network formation is not the result of an earmarked socialization process) to analyze the characteristics of equilibrium networks.

17 All detailed proofs of these robustness results are available upon request.

18 For example, in the model of public goods in networks by Bramoullé and Kranton (2007), the best-reply functions are linear, even though they use a general utility function and not a linear-quadratic one. For an interesting discussion on this issue, see Bramoullé et al. (2009).
} 
(c) On top of its operational virtues, condition (A3) embodies the central assumption of our approach, the genericity of socialization efforts, not earmarked to particular targets. We can extend condition (A3) by, for example, introducing productivity $b_{i}$ in the link formation process so that the ratio $g_{j i}(\mathbf{s}) / g_{k i}(\mathbf{s})$ is not only a function of $s_{j} / s_{k}$ but also of $b_{j} / b_{k}$. Again, in this extension, our main results are still valid.

Our model has, however, some limits. For example, if we consider a model where there is no congestion in social interactions, i.e. $g_{i j}(\mathbf{s})=s_{i} s_{j}$, then the analysis becomes very difficult. Indeed, when there is no congestion in the socialization process, then for large population $n$, the condition on invertibility of the socialization matrix $\mathbf{M}$ will not be satisfied. This is because, when there is no congestion, benefits from socialization explode when $n$ is large. There are, in a sense, "too many" synergies from friends.

We have also illustrated our model in terms of parental involvement in education and derive interesting empirical predictions. We find, in particular, that high-educated parents tend to participate more in social activities related to schools (like e.g. parental evenings) than low-educated parents and that different levels of parental education affect (positively or negatively) proportionally more the socialization of those parents than their direct effort with their children.

\section{Appendix A}

Proof of Lemma 1. Fix s. Combining (A1) and (A3) gives $s_{k} g_{i j}(\mathbf{s})=s_{j} g_{i k}(\mathbf{s})$. Summing across all $j$ 's and using (A2) gives $g_{i k}(\mathbf{s})=s_{i} s_{k} / \sum_{j=1}^{n} s_{j}$.

Proof of Lemma 2. The equilibrium analysis is clear.

Proof of Theorem 1. It follows from the following Lemmas 3, 4, 5 and 6.

Consider an $m$-replica game involving $n=m t$ players, where $m \geqslant 1$ is fixed for the time being.

Let $\mathbf{G}(\mathbf{s})=\left[g_{i j}(\mathbf{s})\right]_{i, j \in N}$ be the $n$-symmetric adjacency matrix for the network with link intensities in (1).

For all $\alpha \geqslant 0$ and for all $\mathbf{x} \in \mathbb{R}_{+}^{n}$, define $\overline{\mathbf{x}}=\sum_{\tau=1}^{n} x_{\tau} / n, \overline{\mathbf{x}^{2}}=\sum_{\tau=1}^{n} x_{\tau}^{2} / n$, and:

$$
\lambda_{\alpha}(\mathbf{x})=\frac{\alpha \overline{\mathbf{x}}}{\overline{\mathbf{x}}-\alpha \overline{\mathbf{x}^{2}}}
$$

We extend this definition to any non-negative vector in an Euclidean space of arbitrary finite size.

Lemma 3. Let $s \in \mathbb{R}_{+}^{n}, \mathbf{s} \neq \mathbf{0}$ and $\alpha \geqslant 0$ such that $1>\alpha \overline{\mathbf{s}^{2}} / \overline{\mathbf{s}}$. Then, $\mathbf{M}(\mathbf{s})=[\mathbf{I}-\alpha \mathbf{G}(\mathbf{s})]^{-1}$ is a well-defined and non-negative $n$-square matrix, equal to $\mathbf{M}(\mathbf{s})=\mathbf{I}+\lambda_{\alpha}(\mathbf{s}) \mathbf{G}(\mathbf{s})$.

Proof. When $\mathbf{M}(\mathbf{s}) \in \mathbb{R}^{n^{2}}$ is well defined, we have $\mathbf{M}(\mathbf{s})=\sum_{p=0}^{+\infty} \alpha^{p} \mathbf{G}(\mathbf{s})^{p}$. We compute $\mathbf{G}(\mathbf{s})^{p}$. First, note that (we omit $\mathbf{s}$ when there is no confusion):

$$
g_{i j}^{[2]}=\sum_{l=1}^{n} g_{i l} g_{l j}=\frac{s_{i} s_{j}}{n \overline{\mathbf{s}}} \sum_{h=1}^{n} \frac{s_{h}^{2}}{n \overline{\mathbf{s}}}=\frac{\overline{\mathbf{s}^{2}}}{\overline{\mathbf{s}}} g_{i j}, \quad \text { for all } i, j=1, \ldots, n .
$$

By a trivial induction on $p=1,2, \ldots$, we deduce that $g_{i j}^{[p]}=\left(\overline{\mathbf{s}^{2}} / \overline{\mathbf{s}}\right)^{(p-1)} g_{i j}$, for all $i, j$ and all $p \geqslant 1$. Therefore:

$$
\mathbf{M}(\mathbf{s})=[\mathbf{I}-\alpha \mathbf{G}(\mathbf{s})]^{-1}=\mathbf{I}+\sum_{p=1}^{+\infty}\left(\alpha \frac{\overline{\mathbf{s}^{2}}}{\overline{\mathbf{s}}}\right)^{p} \mathbf{G}=\mathbf{I}+\lambda_{\alpha}(\mathbf{s}) \mathbf{G}(\mathbf{s}) .
$$

We know from Debreu and Herstein (1953) that $\mathbf{M}(\mathbf{s})$ is well defined and non-negative if and only if $1>\alpha \rho(\mathbf{G}(\mathbf{s}))$, where $\rho(\mathbf{G}(\mathbf{s})$ ) is the modulus of the largest eigenvalue of $\mathbf{G}(\mathbf{s})$ (see also Theorem 1 in Ballester et al., 2006). Let us show that $\rho(\mathbf{G}(\mathbf{s}))=\overline{\mathbf{s}^{2}} / \overline{\mathbf{s}}$.

First, note that $\overline{\mathbf{s}^{2}} / \overline{\mathbf{s}}$ is an eigenvalue of $\mathbf{G}$ for the eigenvector $\mathbf{s}$. Indeed, $\mathbf{G} \cdot \mathbf{s}=\left(\overline{\mathbf{s}^{2}} / \overline{\mathbf{s}}\right) \mathbf{s}$.

Second, let $\mathbf{x}$ such that $\|\mathbf{x}\|=1$. We have:

$$
\mathbf{G} \cdot \mathbf{X}=\frac{\mathbf{s} \cdot \mathbf{x}}{n \overline{\mathbf{s}}} \mathbf{s}
$$

where $\mathbf{s} \cdot \mathbf{x}=\sum_{i=1}^{n} s_{i} x_{i}$ is the scalar product, with $|\mathbf{s} \cdot \mathbf{x}| \leqslant\|\mathbf{s}\| \times\|\mathbf{x}\| \leqslant\|\mathbf{s}\|$. Therefore, $\|\mathbf{G} \cdot \mathbf{x}\| \leqslant\|\mathbf{s}\|^{2} / n \overline{\mathbf{s}}=\overline{\mathbf{s}^{2}} / \overline{\mathbf{s}}$. Note that, by definition, $\rho(\mathbf{G})=\sup \{\|\mathbf{G} \cdot \mathbf{x}\| /\|\mathbf{x}\|:\|\mathbf{x}\|=1\}$. Altogether, we can conclude that $\rho(\mathbf{G})=\overline{\mathbf{s}^{2}} / \overline{\mathbf{s}}$.

Let now $m^{1}, m^{2}, m^{3}, \ldots$ be an increasing sequence of integers such that $m^{h} \rightarrow+\infty$ as $h \rightarrow+\infty$. Each $h \in \mathbb{N}$ defines a $m^{h}$-replica game involving $n^{h}=m^{h} t$ players. In the $m^{h}$-replica game, there are $m^{h}$ players of each type $\left(b_{1}, \ldots, b_{t}\right)$. In each such game, a profile of strategies is $\left(\mathbf{k}^{h}, \mathbf{s}^{h}\right) \in \mathbb{R}_{+}^{n^{h}} \times \mathbb{R}_{+}^{n^{h}}$. Given a player $i=1, \ldots, n^{h}$, recall that $b_{\tau(i)}$ denotes his type, where $\tau(i) \in T$. 
Lemma 4. Let $\left\{\left(\mathbf{s}^{h}, \mathbf{k}^{h}\right)\right\}_{h \in \mathbb{N}}$ be a sequence of Nash equilibria of the $m^{h}$-replica games such that $c>a \overline{\mathbf{s}^{h 2}} / \overline{\mathbf{s}^{h}}$, for all $h \in \mathbb{N}$. Suppose that the system of equations:

$$
\left\{\begin{array}{l}
{[c-a(\mathbf{b}) s] k=1} \\
s=a(\mathbf{b}) k^{2}
\end{array}\right.
$$

has a solution $(s, k) \in \mathbb{R}_{+}^{2}$ such that $c>a(\mathbf{b})$ s. Then, for all $\varepsilon>0$, there exists some $h_{\varepsilon} \in \mathbb{N}$ such that, for all $h \geqslant h_{\varepsilon}$, we have $\max \left\{\left|k_{i}^{h}-b_{\tau(i)} k\right|,\left|s_{i}^{h}-b_{\tau(i)} s\right|\right\}<\varepsilon$, for all $i=1, \ldots, n^{h}$, where $(s, k)$ is a solution to (19).

Proof. Let $\left\{\left(\mathbf{k}^{h}, \mathbf{s}^{h}\right)\right\}_{h \in \mathbb{N}}$ be a sequence of Nash equilibria such that $c>a\left(\mathbf{s}^{h}\right)$, for all $h$. Let $\operatorname{diag}\left(\mathbf{G}\left(\mathbf{s}^{h}\right)\right)$ be the diagonal matrix with diagonal terms $g_{i i}\left(\mathbf{s}^{h}\right)$ and zero off-diagonal terms. For each $h$, the first-order necessary equilibrium conditions for $k^{h}$ are:

$$
\left[c \mathbf{I}-a \mathbf{G}\left(\mathbf{s}^{h}\right)\right] \cdot \mathbf{k}^{h}+a \operatorname{diag}\left(\mathbf{G}\left(\mathbf{s}^{h}\right)\right) \cdot \mathbf{k}^{h}=\mathbf{b}^{h} .
$$

Using the expression for $\left[\mathbf{I}-\alpha \mathbf{G}\left(\mathbf{s}^{h}\right)\right]^{-1} \in \mathbb{R}^{n^{h^{2}}}$ in Lemma 3 and letting $\alpha=a / c$, we rewrite this first-order necessary equilibrium conditions for $k^{h}$ as:

$$
c \mathbf{k}^{h}+a\left[\mathbf{I}+\lambda_{a / c}\left(\mathbf{s}^{h}\right) \mathbf{G}\left(\mathbf{s}^{h}\right)\right] \cdot \operatorname{diag}\left(\mathbf{G}\left(\mathbf{s}^{h}\right)\right) \cdot \mathbf{k}^{h}=\left[\mathbf{I}+\lambda_{a / c}\left(\mathbf{s}^{h}\right) \mathbf{G}\left(\mathbf{s}^{h}\right)\right] \cdot \mathbf{b}^{h},
$$

where $\mathbf{b}^{h} \in \mathbb{R}_{+}^{n^{h}}$ is defined by $b_{i}^{h}=b_{\tau(i)}$, for all $i=1, \ldots, n^{h}$. In words, the $i$ th coordinate of $\mathbf{b}^{h}$ corresponds to the private returns of player $i$ 's type. Note that the $n^{h}$ coordinates of $\mathbf{b}^{h}$ take $t$ different possible values, $b_{1}, \ldots, b_{t}$, each repeated $m^{h}$ times.

We also compute the first-order necessary equilibrium conditions for $s_{i}^{h}$, which are:

$$
s_{i}^{h}=a k_{i}^{h} \frac{\mathbf{s}^{h} \cdot \mathbf{k}^{h}}{n^{h} \overline{\mathbf{s}}^{h}}-a s_{i}^{h} k_{i}^{h} \frac{\mathbf{s}^{h} \cdot \mathbf{k}^{h}}{\left(n^{h} \overline{\mathbf{s}}^{h}\right)^{2}}-a \frac{s_{i}^{h} k_{i}^{h 2}}{n^{h} \overline{\mathbf{s}}^{h}}+a \frac{s_{i}^{h 2} k_{i}^{h 2}}{\left(n^{h} \overline{\mathbf{s}}^{h}\right)^{2}}, \quad i=1, \ldots, n^{h} .
$$

Given that $c>a \overline{\mathbf{s}^{h 2}} / \overline{\mathbf{s}^{h}}$, for all $h$ and that $n^{h} \rightarrow+\infty$ as $h \rightarrow+\infty$, necessarily, $s_{i}^{h} \in O(1),{ }^{19}$ for all $i=1, \ldots, n^{h}$ and for all $h$. Indeed, suppose that $s_{j}^{h} \in O\left(n^{h^{p}}\right), p>0$, for some $j$. Let then $q>0$ such that $s_{i}^{h} \in O\left(n^{h^{q}}\right), p \geqslant q \geqslant 0$, for all $i$. Then, $a \overline{\mathbf{s}^{h 2}} / \overline{\mathbf{s}^{h}} \in O\left(n^{h^{p}}\right)$, and the inequality $c>a \mathbf{s}^{h 2} / \overline{\mathbf{s}^{h}}$ is violated for large enough $h$.

Given that $s_{i}^{h} \in O(1)$, we have $g_{i j}\left(\mathbf{s}^{h}\right)=s_{i}^{h} s_{j}^{h} /\left(\sum_{l=1}^{n^{h}} s_{l}^{h}\right) \in o(1)^{20}$ when $h \rightarrow+\infty$, for all $i, j=1, \ldots, n^{h}$.

The first-order conditions (20) imply that $k_{i}^{h} \in O(1)$, for all $i=1, \ldots, n^{h}$ and for all $h$.

Then, using (21), we deduce that for $h$ high enough, we have

$$
s_{i}^{h}=a k_{i}^{h} \frac{\mathbf{s}^{h} \cdot \mathbf{k}^{h}}{n^{h} \overline{\mathbf{s}^{h}}}+o(1), \quad \text { for all } i=1, \ldots, n^{h} \text { and for all } h .
$$

By (20), $k_{i}^{h}$ is a continuous function of $\mathbf{s}^{h}$. Therefore, $s_{i}^{h}=\sigma_{i}^{h}+o(1)$ and $k_{i}^{h}=\kappa_{i}^{h}+o(1)$, for all $i=1, \ldots, n^{h}$ and for all $h$, where $\left(\boldsymbol{\sigma}^{h}, \boldsymbol{\kappa}^{h}\right)$ are such that:

$$
c \boldsymbol{\kappa}^{h}=\left[\mathbf{I}+\lambda_{a / c}\left(\boldsymbol{\sigma}^{h}\right) \mathbf{G}\left(\boldsymbol{\sigma}^{h}\right)\right] \cdot \mathbf{b}^{h}
$$

and

$$
\sigma_{i}^{h}=a \kappa_{i}^{h} \frac{\boldsymbol{\sigma}^{h} \cdot \boldsymbol{\kappa}^{h}}{n^{h} \overline{\boldsymbol{\sigma}^{h}}}, \quad i=1, \ldots, n^{h} .
$$

We solve for (23) and (24).

Note, first, that (24) implies that $\sigma_{i}^{h} / \kappa_{i}^{h}=\sigma_{j}^{h} / \kappa_{j}^{h}$, for all $i, j$. Without any loss of generality, we can thus write $\sigma_{i}^{h}=\theta_{i}^{h} s$ and $\kappa_{i}^{h}=\theta_{i}^{h} k$, for all $i=1, \ldots, n^{h}$ and for some $k, s$. Then, (24) can be rewritten as:

$$
s=a k^{2} \frac{\overline{\theta^{h 2}}}{\overline{\boldsymbol{\theta}^{h}}} \text {. }
$$

\footnotetext{
$19 f(h)=O(g(h))$ if and only if $\exists M>0, \exists h_{0}>0$ such that $\forall h>h_{0}$, we have $0 \leqslant f(h)<M g(h)$. In other words, $f(h)=O(g(h))$ means that $g(h)$ bounds $f(h)$ from above (for large $h$ ) up to a constant.

$20 f(h)=o(g(h))$ if and only if $\forall M>0, \exists h_{0}>0$ such that $\forall h>h_{0}$, we have $0 \leqslant f(h)<M g(h)$. In other words, $f(h)$ becomes insignificant relative to $g(h)$ as $h$ approaches infinity: $\lim _{h \rightarrow+\infty}[f(h) / g(h)]=0$.
} 
Note also that $g_{i j}\left(\boldsymbol{\sigma}^{h}\right)=\theta_{i}^{h} \theta_{j}^{h} s / n^{h} \overline{\boldsymbol{\theta}^{h}}$ and that $\lambda_{a / c}\left(\boldsymbol{\sigma}^{h}\right)=a \overline{\boldsymbol{\theta}^{h}} /\left(c \overline{\boldsymbol{\theta}^{h}}-a s \overline{\boldsymbol{\theta}^{h 2}}\right)$. We thus rewrite (23) as:

$$
c \theta_{i}^{h} k=b_{i}^{h}+\frac{a s}{n^{h}} \frac{\theta_{i}^{h}}{c \overline{\boldsymbol{\theta}^{h}}-a s \overline{\boldsymbol{\theta}^{h 2}}} \sum_{j=1}^{n^{h}} \theta_{j}^{h} b_{j}^{h}, \quad i=1, \ldots, n^{h} .
$$

From Eq. (26)

$$
\theta_{i}^{h}\left(c k-\frac{a s}{n^{h}} \frac{\sum_{j=1}^{n^{h}} \theta_{j}^{h} b_{j}^{h}}{c \overline{\boldsymbol{\theta}^{h}}-a s \overline{\boldsymbol{\theta}^{h 2}}}\right)=b_{i}^{h}, \quad i=1, \ldots, n^{h},
$$

which implies that for all $i, j$ we must have

$$
\frac{\theta_{i}^{h}}{\theta_{j}^{h}}=\frac{b_{i}^{h}}{b_{j}^{h}}
$$

and hence, w.l.o.g. $\theta_{i}^{h}=b_{i}^{h}$. Then (26) becomes:

$$
c k=1+\frac{a s \overline{\mathbf{b}^{2}}}{c \overline{\mathbf{b}}-a s \overline{\mathbf{b}^{2}}}=\frac{c}{c-a(\mathbf{b}) s},
$$

equivalent to

$$
k=\frac{1}{c-a(\mathbf{b}) s},
$$

while (25) becomes $s=a(\mathbf{b}) k^{2}$.

Finally, note that the condition $c>a \overline{\mathbf{s}^{h 2}} / \overline{\mathbf{s}^{h}}$ is then equivalent to $c>a(\mathbf{b}) s$.

Lemma 5. If $2(c / 3)^{3 / 2}>a(\mathbf{b})$, then the system of Eqs. (19) has exactly two solutions $(s, k) \in \mathbb{R}_{+}^{2}$ such that $c>a(\mathbf{b}) s$.

Proof. Plugging the expression for $k$ into the expression for $s$ in $(19)$, one concludes that every solution $\left(s^{*}, k^{*}\right)$ of $(19)$ is such that $g\left(s^{*}\right)=s^{*}$ where:

$$
g(s)=\frac{a(\mathbf{b})}{[c-a(\mathbf{b}) s]^{2}} .
$$

We establish conditions such that the graph of $g(s)$ crosses (twice) the 45 degree line for some $s$ such that $c>a(\mathbf{b}) s$. Note that $g(0)=a(\mathbf{b}) / c^{2}$ and $\lim _{s \uparrow c / a(\mathbf{b})} g(s)=+\infty$, so that the function $g(\cdot)$ maps $[0, c / a(\mathbf{b}))$ into $\left[a(\mathbf{b}) / c^{2},+\infty\right)$. We have:

$$
g^{\prime}(s)=\frac{2 a(\mathbf{b})^{2}}{[c-a(\mathbf{b}) s]^{3}} .
$$

Therefore, $g^{\prime}(0)=2 a(\mathbf{b})^{2} / c^{3}$ and $\lim _{s \uparrow c / a(\mathbf{b})} g^{\prime}(s)=+\infty$. If there exists a tangent to the graph of $g(\cdot)$ on $[0, c / a(\mathbf{b}))$ which is parallel to the 45 degree line, and if this tangent is strictly below (resp. tangent to) the 45 degree line, the system (19) has exactly two solutions (resp. one solution) on $[0, c / a(\mathbf{b}))$. Such a tangent exists if $2 a(\mathbf{b})^{2} \leqslant c^{3}$, equivalent to $a(\mathbf{b}) \leqslant 2^{-1 / 2} c^{3 / 2}$.

Next, we solve:

$$
g^{\prime}\left(x^{*}\right)=1 \quad \Leftrightarrow \quad a(\mathbf{b}) x^{*}=c-2^{1 / 3} a(\mathbf{b})^{2 / 3} .
$$

Thus, (19) has two solutions (resp. one solution) if and only if $a(\mathbf{b}) \leqslant 2^{-1 / 2} c^{3 / 2}$ and $g\left(x^{*}\right)<x^{*}$ (resp. $\left.g\left(x^{*}\right)=x^{*}\right)$, where $x^{*}$ is defined by (28). We have:

$$
g\left(x^{*}\right)=\frac{a(\mathbf{b})}{\left(2 a(\mathbf{b})^{2}\right)^{2 / 3}}=\frac{a(\mathbf{b})^{-1 / 3}}{2^{2 / 3}} .
$$

Therefore,

$$
g\left(x^{*}\right)<x^{*} \Leftrightarrow a(\mathbf{b})<2 c^{3 / 2} / 3 \sqrt{3} .
$$

The overall condition is thus $a(\mathbf{b})<c^{3 / 2} \min \left\{2 / 3 \sqrt{3}, 2^{-1 / 2}\right\}$. However, it is readily checked that $2 / 3 \sqrt{3}<2^{-1 / 2}$ (indeed, this is equivalent to $2 \sqrt{2}<3 \sqrt{3}$ ). When $a(\mathbf{b})<2(c / 3)^{3 / 2}$ (resp. $a(\mathbf{b})=2(c / 3)^{3 / 2}$ ), the graph of $g(\cdot)$ thus crosses the 45 degree line twice (resp. once) on $[0, c / a(\mathbf{b}))$. 
Lemma 6. Let $\left\{\left(\mathbf{s}^{h *}, \mathbf{k}^{h *}\right)\right\}_{h \in \mathbb{N}}$ be such that $s_{i}^{h *}=s b_{\tau(i)}$ and $k_{i}^{h *}=k b_{\tau(i)}$, for all $i=1, \ldots, n^{h}$, where $(s, k)$ is some given solution to (19). If $2(c / 3)^{3 / 2}>a(\mathbf{b})$, then there exists some $\bar{h} \in \mathbb{N}$ such that, for all $h \geqslant \bar{h}$, the second-order equilibrium conditions for $\mathbf{u}=\left(u_{1}, \ldots, u_{n^{h}}\right)$ hold at $\left(\mathbf{s}^{* h}, \mathbf{k}^{* h}\right)$.

Proof. First note that Lemma 5 implies that (19) has a solution such that $c>a(\mathbf{b}) k$. Consider this solution. We also know from Lemma 4 that both $k_{i}^{h}, s_{i}^{h} \in O(1)$, for all $i=1, \ldots, n^{h}$. We now compute the cross partial derivatives of $\mathbf{u}$ at $\left(\mathbf{s}^{h *}, \mathbf{k}^{h *}\right)$.

First, we have:

$$
\begin{aligned}
& \frac{\partial u_{i}}{\partial s_{i}}\left(\mathbf{s}^{h *}, \mathbf{k}^{h *}\right)=\sum_{j=1, j \neq i}^{n^{h}}\left(\frac{a s_{j}^{h *} k_{i}^{h *} k_{j}^{h *}}{n^{h} \overline{\mathbf{s}^{h *}}}-\frac{a g_{i j}\left(\mathbf{s}^{h *}\right) k_{i}^{h *} k_{j}^{h *}}{n^{h} \overline{\mathbf{s}^{h *}}}\right)-s_{i}^{h *}, \\
& \frac{\partial u_{i}}{\partial k_{i}}\left(\mathbf{s}^{h *}, \mathbf{k}^{h *}\right)=b_{\tau(i)}+a \sum_{j=1, j \neq i}^{n^{h}} g_{i j}\left(\mathbf{s}^{h *}\right) k_{j}^{h *}-c k_{i}^{h *} .
\end{aligned}
$$

Thus

$$
\begin{aligned}
\frac{\partial^{2} u_{i}}{\partial s_{i}^{2}}\left(\mathbf{s}^{h *}, \mathbf{k}^{h *}\right) & =\sum_{j=1, j \neq i}^{n^{h}}\left(-\frac{a s_{j}^{h *} k_{i}^{h *} k_{j}^{h *}}{\left(n^{h} \overline{\mathbf{s}^{h *}}\right)^{2}}-\frac{a s_{j}^{h *} k_{i}^{h *} k_{j}^{h *}}{\left(n^{h} \overline{\mathbf{s}^{h *}}\right)^{2}}+\frac{2 a g_{i j}\left(\mathbf{s}^{h *}\right) k_{i}^{h *} k_{j}^{h *}}{\left(n^{h} \overline{\mathbf{s}^{h *}}\right)^{2}}\right)-1, \\
\frac{\partial^{2} u_{i}}{\partial s_{i} \partial k_{i}}\left(\mathbf{s}^{h *}, \mathbf{k}^{h *}\right) & =\sum_{j=1, j \neq i}^{n^{h}}\left(\frac{a s_{j}^{h *} k_{j}^{h *}}{n^{h} \overline{\mathbf{s}^{h *}}}-\frac{a g_{i j}\left(\mathbf{s}^{h *}\right) k_{j}^{h *}}{n^{h} \overline{\mathbf{s}^{h *}}}\right), \\
\frac{\partial^{2} u_{i}}{\partial k_{i}^{2}}\left(\mathbf{s}^{h *}, \mathbf{k}^{h *}\right) & =-c .
\end{aligned}
$$

So, for $h$ large enough, we get:

$$
\begin{aligned}
& \frac{\partial^{2} u_{i}}{\partial s_{i}^{2}}\left(\mathbf{s}^{h *}, \mathbf{k}^{h *}\right)=o(1)-1, \\
& \frac{\partial^{2} u_{i}}{\partial s_{i} \partial k_{i}}\left(\mathbf{s}^{h *}, \mathbf{k}^{h *}\right)=o(1)+a k \frac{\overline{\mathbf{b}^{2}}}{\overline{\mathbf{b}}}=o(1)+a(\mathbf{b}) k, \\
& \frac{\partial^{2} u_{i}}{\partial k_{i}^{2}}\left(\mathbf{s}^{h *}, \mathbf{k}^{h *}\right)=-c .
\end{aligned}
$$

The second-order conditions amount to checking that the principal minors of the Hessian matrix

$$
\left[\begin{array}{cc}
\frac{\partial^{2} u_{i}}{\partial s_{i}^{2}} & \frac{\partial^{2} u_{i}}{\partial s_{i} \partial k_{i}} \\
\frac{\partial^{2} u_{i}}{\partial s_{i} \partial k_{i}} & \frac{\partial^{2} u_{i}}{\partial k_{i}^{2}}
\end{array}\right]=\left[\begin{array}{cc}
o(1)-1 & o(1)+a(\mathbf{b}) k \\
o(1)+a(\mathbf{b}) k & -c
\end{array}\right]
$$

have alternating signs.

But the determinant of a matrix is a continuous (polynomial) function of the matrix entries. Given that (31) and (33), are negative, when $h \rightarrow+\infty$, we are thus left to check that the sign of the determinant is positive, that is, $c-a(\mathbf{b})^{2} k^{2}>0$, which is equivalent to $c-a(\mathbf{b}) s>0$, which Lemma 5 shows is true when $2(c / 3)^{3 / 2}>a(\mathbf{b})$.

Proof of Proposition 1. Let $\epsilon>0$. Take $h$ large enough such that Theorem 1 holds for this $\epsilon$. We check stability by looking at the behavior of the gradient system

$$
\begin{aligned}
\frac{\partial k_{i}(t)}{\partial t} & =\frac{\partial u_{i}(\mathbf{s}(t), \mathbf{k}(t))}{\partial k_{i}(t)}, \\
\frac{\partial s_{i}(t)}{\partial t} & =\frac{\partial u_{i}(\mathbf{s}(t), \mathbf{k}(t))}{\partial s_{i}(t)}
\end{aligned}
$$

around the equilibrium points.

Let us first look at the partially corner equilibrium. By (29) we have that the first derivative with respect to $k_{i}$ when $h$ is large is

$$
o(1)+\sum_{j=1, j \neq i}^{n^{h}} \frac{a s_{j}^{h *} k_{i}^{h *} k_{j}^{h *}}{n^{h} \overline{\mathbf{s}^{h *}}}-s_{i}^{h *} .
$$


Consider a perturbation around the equilibrium $s^{\varepsilon}=\left(\varepsilon_{1}, \ldots, \varepsilon_{n}\right)$, with $\underline{b}=\min \left\{b_{1}, \ldots, b_{n}\right\}$. Then, the first derivative with respect to $s_{i}$ is approximately

$$
a b_{i} \frac{\sum_{j=1}^{n} \varepsilon_{j} b_{j}}{\sum_{j=1}^{n} \varepsilon_{j}}-\varepsilon_{i}>a b_{i} \underline{b}-\varepsilon_{i}>0,
$$

for $\varepsilon_{i}$ small enough. For any small enough perturbation, $s_{i}$ would tend to increase for all $i$, thus negating stability.

If we linearize the dynamic system (34), (35) around the equilibria we get, for all $i=1, \ldots, n^{h}$ :

$$
\begin{aligned}
\frac{\partial k_{i}(t)}{\partial t} & =\sum_{j=1}^{n^{h}} \frac{\partial^{2} u_{i}}{\partial k_{i} \partial k_{j}}\left(\mathbf{s}^{h *}, \mathbf{k}^{h *}\right)\left(k_{j}(t)-k_{j}^{*}\right)+\sum_{j=1}^{n^{h}} \frac{\partial^{2} u_{i}}{\partial k_{i} \partial s_{j}}\left(\mathbf{s}^{h *}, \mathbf{k}^{h *}\right)\left(s_{j}(t)-s_{j}^{*}\right), \\
\frac{\partial s_{i}(t)}{\partial t} & =\sum_{j=1}^{n^{h}} \frac{\partial^{2} u_{i}}{\partial s_{i} \partial s_{j}}\left(\mathbf{s}^{h *}, \mathbf{k}^{h *}\right)\left(s_{j}(t)-s_{j}^{*}\right)+\sum_{j=1}^{n^{h}} \frac{\partial^{2} u_{i}}{\partial s_{i} \partial k_{j}}\left(\mathbf{s}^{h *}, \mathbf{k}^{h *}\right)\left(k_{j}(t)-k_{j}^{*}\right) .
\end{aligned}
$$

For $i \neq j$, we have:

$$
\begin{aligned}
\frac{\partial^{2} u_{i}}{\partial s_{i} \partial s_{j}}\left(\mathbf{s}^{h *}, \mathbf{k}^{h *}\right) & =\frac{a k_{i}^{h *} k_{j}^{h *}}{n^{h} \overline{\mathbf{s}^{h *}}}-\frac{a s_{i}^{h *} k_{i}^{h *} k_{j}^{h *}}{\left(n^{h} \overline{\mathbf{s}^{h *}}\right)^{2}}+\sum_{l=1, l \neq i}^{n^{h}}\left(-\frac{a s_{l}^{h *} k_{i}^{h *} k_{l}^{h *}}{\left(n^{h} \overline{\mathbf{s}^{h *}}\right)^{2}}+\frac{a g_{i l}\left(\mathbf{s}^{h *}\right) k_{i}^{h *} k_{l}^{h *}}{\left(n^{h} \overline{\mathbf{s}^{h *}}\right)^{2}}\right), \\
\frac{\partial^{2} u_{i}}{\partial s_{i} \partial k_{j}}\left(\mathbf{s}^{h *}, \mathbf{k}^{h *}\right) & =\frac{a s_{j}^{h *} k_{i}^{h *}}{n^{h} \overline{\mathbf{s}^{h *}}}-\frac{a g_{i j}\left(\mathbf{s}^{h *}\right) k_{i}^{h *}}{n^{h} \overline{\mathbf{s}^{h *}}}, \\
\frac{\partial^{2} u_{i}}{\partial k_{i} \partial s_{j}}\left(\mathbf{s}^{h *}, \mathbf{k}^{h *}\right) & =\frac{a s_{i}^{h *} k_{j}^{h *}}{n^{h} \overline{\mathbf{s}^{h *}}}-\frac{a g_{i j}\left(\mathbf{s}^{h *}\right) k_{j}^{h *}}{n^{h} \overline{\mathbf{s}^{h *}}}, \\
\frac{\partial^{2} u_{i}}{\partial k_{i} \partial k_{j}}\left(\mathbf{s}^{h *}, \mathbf{k}^{h *}\right) & a g_{i j}\left(\mathbf{s}^{h *}\right) .
\end{aligned}
$$

Thus, we have when $h$ gets large and for $i \neq j$ :

$$
\begin{aligned}
\frac{\partial^{2} u_{i}}{\partial s_{i} \partial s_{j}}\left(\mathbf{s}^{h *}, \mathbf{k}^{h *}\right) & =o(1), \\
\frac{\partial^{2} u_{i}}{\partial s_{i} \partial k_{j}}\left(\mathbf{s}^{h *}, \mathbf{k}^{h *}\right) & =o(1), \\
\frac{\partial^{2} u_{i}}{\partial k_{i} \partial s_{j}}\left(\mathbf{s}^{h *}, \mathbf{k}^{h *}\right) & =o(1), \\
\frac{\partial^{2} u_{i}}{\partial k_{i} \partial k_{j}}\left(\mathbf{s}^{h *}, \mathbf{k}^{h *}\right) & =o(1) .
\end{aligned}
$$

The coefficients of the linearized gradient system (36) correspond to the cells of a $2 n^{h} \times 2 n^{h}$ matrix $\boldsymbol{\Pi}^{h}\left(\left(\mathbf{s}^{h *}, \mathbf{k}^{h *}\right)\right)$ which, when $h$ is large enough, gets arbitrarily close to the following matrix $\Pi^{h}$ :

$$
\boldsymbol{\Pi}^{h}=\left[\begin{array}{cc}
\mathbf{A}, & \mathbf{B} \\
\mathbf{B}, & \mathbf{c A}
\end{array}\right]
$$

where A, B are the following $n^{h} \times n^{h}$ diagonal matrices

$$
\mathbf{A}=\left[\begin{array}{ccc}
-1 & \ldots & 0 \\
\ldots & \ldots & \ldots \\
0 & \ldots & -1
\end{array}\right], \quad \mathbf{B}=\left[\begin{array}{ccc}
a(\mathbf{b}) k & \ldots & 0 \\
\ldots & \ldots & \ldots \\
0 & \ldots & a(\mathbf{b}) k
\end{array}\right]
$$

Let $\lambda$ be an eigenvalue of $\boldsymbol{\Pi}^{h}$ for some eigenvector $\boldsymbol{v}_{\lambda}^{\top}=\left[\mathbf{x}_{\lambda}^{h_{\top}}, \mathbf{y}_{\lambda}^{h_{\top}}\right], i=1, \ldots, n^{h}$ where $\mathbf{x}_{\lambda}^{h}$ and $\mathbf{y}_{\lambda}^{h}$ are $n^{h} \times 1$ vectors with coordinates $x_{i, \lambda}^{h}$ and $y_{i, \lambda}^{h}, i=1, \ldots, n^{h}$. The eigenvalue and the eigenvalue satisfy the following identity: $\boldsymbol{\Pi}^{h} \boldsymbol{v}_{\lambda}=\lambda \boldsymbol{v}_{\lambda}$, that is:

$$
\begin{aligned}
& (\lambda+1) x_{i, \lambda}^{h}-a(\mathbf{b}) k y_{i, \lambda}^{h}=0, \quad i=1, \ldots, n^{h}, \\
& -a(\mathbf{b}) k x_{i, \lambda}^{h}+(\lambda+c) y_{i, \lambda}^{h}=0, \quad i=1, \ldots, n^{h} .
\end{aligned}
$$


These systems have a non-trivial solution if and only if $(\lambda+1)(\lambda+c)-(a(\mathbf{b}) k)^{2}=0$. This second-order equation has a discriminant equal to $(c-1)^{2}+4(a(\mathbf{b}) k)^{2} \geqslant 0$. It thus always admits two real solutions, denoted $\lambda^{1}$ and $\lambda^{2}$. Besides:

$$
\lambda^{1}+\lambda^{2}=-(1+c)<0,
$$

implying that at least one such solution is negative. Then, both are negative if and only if $\lambda^{1} \lambda^{2}=c-(a(\mathbf{b}) k)^{2}>0$, equivalent to $c-a(\mathbf{b}) s>0$, which is true when $2(c / 3)^{3 / 2}>a(\mathbf{b})$.

Summing up, when $2(c / 3)^{3 / 2}>a(\mathbf{b})>0$, the matrix $\boldsymbol{\Pi}^{h}$ has two different negative eigenvalues $\lambda^{1}, \lambda^{2}<0$. Each eigenvalue $\lambda^{r}, r=1,2$ has an associated eigenspace of dimension $n^{h}$ with generating eigenvectors $\left[a(\mathbf{b}) k \mathbf{i}^{h \top},\left(\lambda^{r}+1\right) \mathbf{i}^{h \top}\right]$, $i=1, \ldots, n^{h}$ where $\mathbf{i}^{h}$ is an $n^{h} \times 1$ vector containing a 1 in position $i=1, \ldots, n^{h}$ and 0 's in the other $n^{h}-1$ positions.

Proof of Proposition 2. Let $\epsilon>0$. Take $h$ large enough such that Theorem 1 holds for this $\epsilon$, and let ( $\left.\mathbf{s}^{h *}, \mathbf{k}^{h *}\right)$ and $\left(\mathbf{s}^{h * *}, \mathbf{k}^{h * *}\right)$ be the two approximate equilibria, where $s_{i}^{h *}=s^{*} b_{\tau(i)}, k_{i}^{h *}=k^{*} b_{\tau(i)}$, and $s_{i}^{h * *}=s^{* *} b_{\tau(i)}, k_{i}^{h * *}=k^{* *} b_{\tau(i)}$, for all $i=1, \ldots, n^{h}$, and $\left(s^{*}, k^{*}\right),\left(s^{* *}, k^{* *}\right)$ are the two different solutions to (6). Suppose that $s_{i}^{h *} \geqslant s_{i}^{h * *}$, for some $i$. Then, necessarily, $s^{*} \geqslant s^{* *}$. By (6), we deduce that $k^{*} \geqslant k^{* *}$. Therefore, both $s_{i}^{h *} \geqslant s_{i}^{h * *}$ and $k_{i}^{h *} \geqslant k_{i}^{h * *}$, for all $i=1, \ldots, n^{h}$.

To establish the welfare ranking, we first use the expression for payoffs in (4) and the first-order conditions for $k_{i}^{h}$, to obtain the following expression for approximate equilibrium payoffs:

$$
u_{i}^{h *}=\frac{b_{\tau(i)}^{2}}{2}\left(c k^{* 2}-s^{* 2}\right)+o(1), \quad \text { for all } i=1, \ldots, n^{h} .
$$

Next, using the fact that $\left(s^{*}, k^{*}\right)$ are solutions to (6), we write:

$$
c k^{* 2}-s^{* 2}=c \frac{s^{*}}{a(\mathbf{b})}-s^{* 2}=\frac{s^{*}}{a(\mathbf{b})}\left(c-a(\mathbf{b}) s^{*}\right)=\frac{1}{a(\mathbf{b})} \frac{s^{*}}{k^{*}}=k^{*},
$$

and thus:

$$
u_{i}^{h *}=\frac{b_{\tau(i)}^{2}}{2} k^{*}+o(1), \quad \text { for all } i=1, \ldots, n^{h},
$$

and similarly for the approximate equilibrium payoffs $u_{i}^{h * *}$ that correspond to (s $\left.\mathbf{s}^{h * *}, \mathbf{k}^{h * *}\right)$. Since, by definition $k^{*} \geqslant k^{* *}$, the welfare at the equilibrium $\left(\mathbf{s}^{h *}, \mathbf{k}^{h *}\right)$ is higher than at the equilibrium $\left(\mathbf{s}^{h * *}, \mathbf{k}^{h * *}\right)$.

To establish the position of the efficient outcome, the planners' problem is:

$$
\sum_{i=1}^{n}\left(b_{\tau(i)} k_{i}+a \sum_{j=1, j \neq i}^{n} g_{i j}(\mathbf{s}) k_{j} k_{i}-\frac{1}{2} c k_{i}^{2}-\frac{1}{2} s_{i}^{2}\right)
$$

for which FOC's are given by:

$$
\begin{aligned}
& c \mathbf{k}^{h}+2 a\left[\mathbf{I}+\lambda_{a / c}\left(\mathbf{s}^{h}\right) \mathbf{G}\left(\mathbf{s}^{h}\right)\right] \cdot \operatorname{diag}\left(\mathbf{G}\left(\mathbf{s}^{h}\right)\right) \cdot \mathbf{k}^{h}=\left[\mathbf{I}+\lambda_{a / c}\left(\mathbf{s}^{h}\right) \mathbf{G}\left(\mathbf{s}^{h}\right)\right] \cdot \mathbf{b}^{h}, \\
& s_{i}^{h}=2 a k_{i}^{h} \frac{\mathbf{s}^{h} \cdot \mathbf{k}^{h}}{n^{h} \overline{\mathbf{s}}^{h}}-2 a s_{i}^{h} k_{i}^{h} \frac{\mathbf{s}^{h} \cdot \mathbf{k}^{h}}{\left(n^{h} \overline{\mathbf{s}}^{h}\right)^{2}}-2 a \frac{s_{i}^{h} k_{i}^{h 2}}{n^{h} \overline{\mathbf{s}}^{h}}+2 a \frac{s_{i}^{h 2} k_{i}^{h 2}}{\left(n^{h} \overline{\mathbf{s}}^{h}\right)^{2}}, \quad i=1, \ldots, n^{h} .
\end{aligned}
$$

Notice that conditions (43) and (44) are like (21) and (20) except with $2 a$ rather than $a$, and therefore its approximate version is:

$$
\left\{\begin{array}{l}
s=2 a(\mathbf{b}) k^{2} \\
k[c-2 a(\mathbf{b}) s]=1 .
\end{array}\right.
$$

We can thus use the results of proposition elasticity to establish that the (two) solutions of (45) will lie between the two solution of (6). Since the second-order conditions will be satisfied (by a reason analogous to the one in Lemma 6, one of those solutions will be the optimal point). Indeed, by the reasoning in this same proposition the component-wise higher solution is the optimal one. The result now follows.

Proof of Proposition 3. Let $\epsilon>0$. Take $h$ large enough such that Theorem 1 holds for this $\epsilon$. We denote by $\left(\mathbf{s}^{h *}, \mathbf{k}^{h *}\right)$ and $\left(\mathbf{s}^{h * *}, \mathbf{k}^{h * *}\right)$ the corresponding $\epsilon$-equilibria, where $\left(\mathbf{s}^{h *}, \mathbf{k}^{h *}\right) \geqslant\left(\mathbf{s}^{h * *}, \mathbf{k}^{h * *}\right)$ are computed with two different solutions $\left(s^{*}, k^{*}\right) \geqslant\left(s^{* *}, k^{* *}\right)$ of $(19)$. On the $(s, k)$ plane, an increase in $a(\mathbf{b})$ results in an upward shift of the graph of:

$$
k=\frac{1}{c-a(\mathbf{b}) s},
$$

and a downward shift of the graph of:

$$
s=a(\mathbf{b}) k^{2} .
$$


Therefore, the equilibrium actions of the Pareto-inferior equilibrium ( $\left.\mathbf{s}^{h * *}, \mathbf{k}^{h * *}\right)$ all increase, while those of the Paretosuperior equilibrium $\left(\mathbf{s}^{h *}, \mathbf{k}^{h *}\right)$ all decrease. The elasticity $\eta$ that keeps track of the relative changes on $s$ and $k$ when $a(\mathbf{b})$ varies is:

$$
\eta=\frac{s}{k} \frac{\frac{\partial k}{\partial a(\mathbf{b})}}{\frac{\partial s}{\partial a(\mathbf{b})}} .
$$

Differentiating (46) and (47) with respect to $a(\mathbf{b})$ gives:

$$
\begin{aligned}
& \frac{\partial k}{\partial a(\mathbf{b})}=s k^{2}+a(\mathbf{b}) k^{2} \frac{\partial s}{\partial a(\mathbf{b})}, \\
& \frac{\partial s}{\partial a(\mathbf{b})}=k^{2}+2 \frac{s}{k} \frac{\partial k}{\partial a(\mathbf{b})} .
\end{aligned}
$$

Solving for the two partial derivatives gives:

$$
\begin{aligned}
\frac{\partial k}{\partial a(\mathbf{b})} & =\frac{2 s k^{2}}{1-2 a(\mathbf{b}) s k}, \\
\frac{\partial s}{\partial a(\mathbf{b})} & =\frac{k^{2}}{1-2 a(\mathbf{b}) s k}\left(2 \frac{s^{2}}{k}+1\right)
\end{aligned}
$$

and thus:

$$
\eta=\frac{s}{k} \frac{\frac{\partial k}{\partial a(\mathbf{b})}}{\frac{\partial s}{\partial a(\mathbf{b})}}=\frac{2 s^{2}}{k+2 s^{2}}<1
$$

Proof of Proposition 5. It is readily checked that $\partial a(\mathbf{b}) / \partial b_{\tau}>0$ if and only if $b_{\tau}^{2}+2 b_{\tau} \sum_{\tau^{\prime} \neq \tau} b_{\tau^{\prime}} \geqslant 2 \sum_{\tau^{\prime} \neq \tau} b_{\tau^{\prime}}^{2}$, for all $\tau \in T$, a sufficient condition for which is $2 b_{1} \geqslant b_{t}$.

Proof of Proposition 6. Let $\epsilon>0$. Take $h$ large enough such that Theorem 1 holds for this $\epsilon$. We denote by (s $\left.{ }^{h *}, \mathbf{k}^{h *}\right)$ and $\left(\mathbf{s}^{h * *}, \mathbf{k}^{h * *}\right)$ the corresponding $\epsilon$-equilibria, where $\left(\mathbf{s}^{h *}, \mathbf{k}^{h *}\right) \geqslant\left(\mathbf{s}^{h * *}, \mathbf{k}^{h * *}\right)$ are computed with two different solutions $\left(s^{*}, k^{*}\right) \geqslant\left(s^{* *}, k^{* *}\right)$ of $(6)$

Using (42) and (6), and summing across all players gives the following expression for the population average equilibrium payoffs:

$$
\sum_{i=1}^{n^{h}} u_{i}^{h *}=\frac{1}{n^{h}} \sum_{i=1}^{n^{h}} \frac{b_{\tau(i)}^{2}}{2 a(\mathbf{b})} \frac{s^{*}}{k^{*}}+o(1)=\frac{1}{a t} \frac{s^{*}}{k^{*}} \sum_{\tau=1}^{t} b_{\tau}+o(1) .
$$

Following a mean preserving spread in individual traits, $a(\mathbf{b})$ increases and we can conclude.

\section{References}

Ballester, Coralio, Calvó-Armengol, Antoni, forthcoming. Interactions with hidden complementarities. Reg. Sci. Urban Econ., doi: $10.1016 / j . r e g s c i u r b e c o$. 2010.04.003.

Ballester, Coralio, Calvó-Armengol, Antoni, Zenou, Yves, 2006. Who's who in networks? Wanted: the key player. Econometrica 74, $1403-1417$.

Becker, Gary, 1974. A theory of social interactions. J. Polit. Economy 82, 1063-1093.

Bisin, Alberto, Verdier, Thierry, 2000. Beyond the melting pot: Cultural transmission, marriage, and the evolution of ethnic and religious traits. Quart. J. Econ. 115, 955-988.

Bloch, Francis, Dutta, Bhaskar, 2009. Communication networks with endogenous link strength. Games Econ. Behav. 66, 39-56. Bramoullé, Yann, Kranton, Rachel, 2007. Public goods in networks. J. Econ. Theory 135, 478-494.

Bramoullé, Yann, Kranton, Rachel, D’Amours, Martin, 2009. Strategic interaction and networks. Duke University. Unpublished manuscript. Calvó-Armengol, Antoni, Ilkiliç, Rahmi, 2009. Pairwise stability and Nash equilibria in network formation. Int. J. Game Theory 38, 51-79.

Coleman, James S., 1990. Foundations of Social Theory. Harvard Univ. Press, Cambridge.

Corchón, Luis C., Mas-Colell, Andreu, 1996. On the stability of best reply and gradient systems with applications to imperfectly competitive models. Econ. Letters 51, 59-65.

Debreu, Gérard, Herstein, Israel Nathan, 1953. Nonnegative square matrices. Econometrica 21 (4), 597-607.

Dee, Thomas S., 2004. Are there civic returns to education? J. Public Econ. 88, 1197-1720.

Durlauf, Steven N., 2002. On the empirics of social capital. Econ. J. 112, F459-F479.

Galeotti, A. Merlino, L., 2009. Endogenous job contact networks. University of Essex. Unpublished manuscript.

Glaeser, Edward L., Sacerdote, Bruce, Scheinkman, José A., 1996. Crime and social interactions. Quart. J. Econ. 111, 507-548.

Golub, B., Livne, Y., 2010. Strategic random networks. Why social networking technology matters. Stanford University. Unpublished manuscript.

Goyal, Sanjeev, 2007. Connections: An Introduction to the Economics of Networks. Princeton Univ. Press, Princeton.

Helliwell, John F., Putnam, Robert D., 2007. Education and social capital. Eastern Econ. J. 33, 1-19.

Ioannides, Yannis M., Soetevent, Adriaan R., 2007. Social networking and individual outcomes beyond the mean field case. J. Econ. Behav. Organ. 64, 369-390. 
Jackson, Matthew O., 2008. Social and Economic Networks. Princeton Univ. Press, Princeton.

Jackson, Matthew O., Wolinsky, Asher, 1996. A strategic model of social and economic networks. J. Econ. Theory 71, 44-74.

Milligan, Kevin, Moretti, Enrico, Oreopoulos, Philip, 2004. Does education improve citizenship? Evidence from the United States and the United Kingdom. J. Public Econ. 88, 1167-1196.

Patacchini, Eleonora, Zenou, Yves, 2010. Intergenerational education transmission: neighborhood quality and/or parents' involvement? Stockholm University. Unpublished manuscript.

Putnam, Robert D., 1995. Tuning in, tuning out: the strange disappearance of social capital in America. Amer. Prospect 24, 664-683.

Putnam, Robert D., 2000. Bowling Alone: The Collapse and Revival of American Community. Simon and Schuster, New York.

Scheinkman, José A., 2008. Social interactions. In: Durlauf, S., Blume, L. (Eds.), The New Palgrave Dictionary of Economics. 2nd edition. Palgrave Macmillan.

Sobel, Joel, 2002. Can we trust social capital? J. Econ. Lit. 40, 139-154.

Vega-Redondo, Fernando, 2007. Complex Social Networks. Econometric Society Monograph Series. Cambridge Univ. Press, Cambridge. 